\title{
Metastable Dynamics above the Glass Transition
}

\author{
Joonhyun Yeo and Gene F. Mazenko \\ The James Franck Institute and Department of Physics \\ The University of Chicago, Chicago, Illinois 60637
}

(June 16, 2021)

\begin{abstract}
The element of metastability is incorporated in the fluctuating nonlinear hydrodynamic description of the mode coupling theory (MCT) of the liquidglass transition. This is achieved through the introduction of the defect density variable $n$ into the set of slow variables with the mass density $\rho$ and the momentum density g. As a first approximation, we consider the case where motions associated with $n$ are much slower than those associated with $\rho$. Selfconsistently, assuming one is near a critical surface in the MCT sense, we find that the observed slowing down of the dynamics corresponds to a certain limit of a very shallow metastable well and a weak coupling between $\rho$ and $n$. The metastability parameters as well as the exponents describing the observed sequence of time relaxations are given as smooth functions of the temperature without any evidence for a special temperature. We then investigate the case where the defect dynamics is included. We find that the slowing down of the dynamics corresponds to the system arranging itself such that the kinetic coefficient $\gamma_{v}$ governing the diffusion of the defects approaches from above a small temperature-dependent value $\gamma_{v}^{c}$.
\end{abstract}

64.70.Pf, 64.60.My, 66.30.Lw

Typeset using REVTEX 


\section{INTRODUCTION}

While there has been considerable recent progress in the development of the theory of relaxation near the liquid-glass transition, these theories have ignored one of the fundamental defining qualities of the problem: These systems are metastable. In this paper, we introduce the element of metastability into a framework that connects with mode coupling theory (MCT) [1]. We find a theoretical picture which is closer to the observed experimental picture than conventional MCT, since it is freed from the idea of a sharp transition temperature $T_{0}$.

The dynamics of supercooled liquids is characterized by very long relaxation times and a rapid increase of the viscosity with decreasing temperature. Thermodynamic quantities such as specific heat, compressibility, etc. show a change when the temperature reaches the so-called calorimetric glass transition temperature $T_{g}$ which is defined somewhat arbitrarilty by the temperature where the viscosity reaches $\sim 10^{15}$ poise. Recent interests, however, have focused on the study of the relaxation of supercooled liquids at temperatures well above $T_{g}$. The reason for this focus has been mainly due to the discovery of the existence of a very elaborate sequence of time relaxations spread over many decades of time. This sequence has been observed in a series of experiments including light scattering [2,3], neutron scattering [4] and dielectric measurements [5,6]. As depicted for the density auto-correlation function $\phi(t)$ in Fig. 1, this sequence of time relaxations is a three-step process with characterizing exponents, $a, b$ and $\beta$. After a fast microscopic process, $\phi(t)$ decays as a power law, $f+A_{1} t^{-a}$ followed by the so-called von-Schweidler relaxation, $f-A_{2} t^{b}$. For longer times $t>\tau_{\alpha}, \phi(t)$ decays with a stretched exponential, $\exp \left\{-\left(t / \tau_{\alpha}\right)^{\beta}\right\}, \beta<1$.

The mode coupling theory has been very successful in explaining this sequence of time relaxations. As originally formulated by Leutheusser [7], MCT is based on the nonlinear feedback mechanism where the renormalized viscosity is expressed in terms of the integral of the quadratic term in density auto-correlation functions. If we ignore the coupling of the

density to the energy density, the Fourier-Laplace transform of $\phi(t)$ can be written in the generalized-hydrodynamics form [8] 


$$
\phi(z)=\frac{z+i \Gamma(z)}{z^{2}-\Omega_{0}^{2}+i z \Gamma(z)},
$$

where $\Omega_{0}$ is a microscopic 'phonon' frequency and $\Gamma(z)$ is the renormalized viscosity and the wavenumber dependence has been suppressed. In MCT [9] it is assumed that $\Gamma(z)$ can be expressed in the form

$$
\Gamma(z)=d_{0}+\Omega_{0}^{2} \int_{0}^{\infty} d t e^{i z t} \sum_{n=1}^{N} c_{n} \phi^{n}(t),
$$

where $d_{0}$ is the bare viscosity. The original theory due to Leutheusser [7] corresponds to all $c_{n}=0$ except $c_{2}$. This theory does not lead to the von-Schweidler regime and does not give stretched dynamics. Instead, one finds the exponential decay $(\beta=1)$ following the power law decay regime. The importance of the term linear in $\phi(t)\left(c_{1}\right)$ in $\Gamma(z)$ was emphasized by Götze [10] as a sufficient condition for obtaining the stretched exponential relaxation and von-Schweidler regime.

It was first noticed in Ref. [8] that MCT can be understood in terms of the fluctuating nonlinear hydrodynamics $(\mathrm{FNH})$. This analysis was then formulated in detail by Das and Mazenko [11]. One can then use a well-developed field-theoretic methods [12,13] to determine $\Gamma(z)$ perturbatively for almost any choice of the free energy. In this formalism, it can be understood [14], for example, how the linear term in $\Gamma(z)$ is generated. Götze had originally introduced the $c_{1}$ term on phenomenological grounds [10].

Despite its success, the current status of MCT is not without questions and controversies. First of all, the scaling picture arising from the experiments of Dixon et al. [6] shows universal features beyond those suggested by MCT. In their dielectric measurements, they were able to find a scaling curve that fits all the data obtained over 13 decades of frequency range for various glass forming liquids and for different temperatures. This beautiful result has not yet found an explanation within MCT. As indicated by Kim and Mazenko [15], the data of Ref. [6] also implies a universal relationship between the von-Schweidler exponent $b$ and the stretching exponent $\beta$ : $(1+b) /(1+\beta)=$ universal constant. Although this result can be made compatible with MCT [15], it requires the introduction of additional arbitrary constraints. 
Another important question associated with MCT concerns the temperature dependence of the theory. Conventional MCT [1, 1, 10, 16] views the glass transition as a sharp ergodicnonergodic transition as the temperature $T$ approaches the ideal glass transition temperature $T_{0}$ well above $T_{g}$. According to conventional MCT, the relaxation sequence occurs only near $T \sim T_{0}$ and in particular the von-Schweidler relaxation and the stretching are confined to the region $T>T_{0}$. This sharp temperature dependence is clearly in contrast with the universal behavior of Dixon et al. whose data cover any reasonable choice of $T_{0}$. Also, as shown in other recent experiments [17], the exisistence of a well-defined temperature $T_{0}$ well above $T_{g}$ is very doubtful. In this formulation of MCT, the exponents governing the time relaxations are temperature independent just as those involved in a second order phase transition. This is again in disagreement with recent experiments. In particular, Dixon et al. finds $\beta$ weakly temperature dependent, which means, by virtue of the universal relationship mentioned above, that $b$ also depends on temperature. Since MCT predicts [16] that the exponents $a$ and $b$ are related through a temperature-independent relation, this also implies that $a$ is temperature dependent.

Das and Mazenko [11], in their study of FNH of dense fluids, discovered that there exists an important nonhydrodynamic correction that cuts off the sharp nature of the ideal glass transition. This cutoff corresponds to an extra contribution $\gamma(z)$ in Eq. (1.1):

$$
\phi(z)=\frac{z+i \Gamma(z)}{z^{2}-\Omega_{0}^{2}+i \Gamma(z)[z+i \gamma(z)]}
$$

Although it is now widely believed that the sharp transition is indeed smeared by this cutoff mechanism, the question of the exisistence of $T_{0}$ still remains. There exist recent efforts [18 to reconcile the discrepancies between experiments and MCT, which includes the cutoff effect into conventional MCT but still assumes a rounded transition around $T \sim T_{0}$. This attempt, however, ends up with adjusting as many as seven independent parameters to fit experimental data. A simpler interpretation of the situation is that MCT, despite all the successes, needs to be reformulated at a more fundamental level such that it is not tied to the notion of a sharp transition temperature. 
In Ref. [19], we proposed a model where defects and metastabilty play an important role in the glass transition in an attempt to reformulate MCT such that it is compatible with experiments. Although this new theory does not provide any clear explanation for the scaling result of Ref. [6], it does result in a smooth temperature variation without any indication of a special temperature $T_{0}$. The various exponents, according to the model, are weak functions of temperature. In this paper, our main focus is to elaborate on the detailed construction of the model, which was omitted in Ref. [19], and to further elaborate on the dynamics of the defects which we treated using a simplifying assumption in the previous work.

The model introduced in Ref. [19 is based on the introduction of the defect variable $n$ into the set of slow variables consisting of the mass density $\rho$, and the momentum density $\mathbf{g}$, in the FNH description of MCT [11]. The introduction of the defect variable would be required in a rigorous hydrodynamic description of crystalline solids, along with the NambuGoldstone modes associated with broken translational symmetry [20]. Although there is no broken continuous symmetry involved in the glass transition, we consider the situation where this variable plays a role in the transition [21]. The best analogy to the present case would be the situation where one considers an order parameter in the disordered state. We do not need a microscopic definition for the defect variable here. The only information we need here is that the defect density has the usual Poisson bracket relations of a scalar variable with the momentum density $\mathbf{g}$, that they are metastable and that they interact weakly with the mass density.

One of the key assumptions in the model is that motions associated with the defect density $n$ have a very long time scale compared to that of density fluctuation. This is realized in the model via an explicit double well potential $h(n)$ for the defect variable with the metastable defect density $\bar{n}$ associated with the minimum of the higher well. A very small diffusion coefficient $\Gamma_{v}$ for $n$ results from a rolling around in a shallow metastable well. We find that the coupling between the mass density and the defect variables slows down the defect motion further. The coupling also enables the slow dynamics of defects to influence 
the density dynamics. Over the significant time period where the defect is trapped within the metastable well, the defect auto-correlation function $\psi(t)$ can be regarded as a constant while the density auto-correlation function $\phi(t)$ displays the relaxation sequence. This is essentially the basic mechanism discussed in Ref. [14] that generates the linear term in the mode coupling integral. Since one variable (in this case the defect variable) is extremely slow compared to the other, we can replace a term like $\psi(t) \phi(t)$ in $\Gamma(z)$ by a constant times $\phi(t)$. Under this assumption, we find that the observed stretched dynamics corresponds to a certain self-consistent limit of weak coupling and low activation barrier for the defect. As will be discussed below, this limit corresponds to the situation where the coupling energy is weak enough not to destroy the metastable defects but still strong enough for the slow dynamics of defects to result in the slowing down of the mass density variable. In this limit, the parameters describing the double-well potential and the coupling, as well as the exponents of the relaxation sequence are self-consistently determined as smooth functions of temperature.

Since the defect variable is diffusive, the defect auto correlation function $\psi(t)$ decays, to linear order, as $\sim \exp \left(-\gamma_{v} t\right)$, where in terms of the bare diffusion coefficient $\Gamma_{v}$ for $n, \gamma_{v}=h^{\prime \prime}(\bar{n}) \Gamma_{v} q^{2}$ at the wavenumber $q$. Self-consistently we find, using the previous assumption that $\psi(t)$ is regarded as a constant, which corresponds to taking the limit $\gamma_{v} \rightarrow 0$, that $h^{\prime \prime}(\bar{n})$ must be small, i.e. the metastable wells become very shallow and broad. As one moves into the later stage of relaxation, however, we expect that nonlinear corrections to $\gamma_{v}$ become important. Therefore, we need to consider the dynamics of the defects and their coupling to the density fluctuations by including the renormalization of $\gamma_{v}$ in the evolution equation for $\psi(t)$. In this case, $\Gamma(z)$ in Eq. (1.1) is not represented simply by the equation for $\phi(t)$ alone as in Eq. (1.2), since the $c_{1}$ term is now written as $\psi(t) \phi(t)$ with a nonconstant $\psi(t)$. Instead, we obtain a set of coupled equations for $\phi(t)$ and $\psi(t)$ governing the dynamics of both density and defect variables.

We find, through mainly numerical investigations, that the extended model including the defect dynamics, provides considerable self-consistent information on the nature of the 
defects near the glass transition. In particular, we find that the bare value of $\gamma_{v}$ is significantly restricted when the observed stretching occurs. In fact the long time scale of the defect variable corresponds to the case where the system arranges itself such that $\gamma_{v}$ is close to some small temperature-dependent value $\gamma_{v}^{c}$. The system seems to pick out its own defect potential and diffusion coefficient. We find that, when $\gamma_{v} \simeq \gamma_{v}^{c}$, the time scale associated with the defects become much longer than that of the density variable, and thus the basic picture obtained using the previous simplifying assumption still holds: The parameters describing the metastable wells and the coupling arrange themselves such that the coupling enables the slow dynamics of defects to effect the density dynamics without destroying the metastable wells.

We note that the approach we take in this paper is not to explain how the defects bring the system to the observed slowing down starting from the microscopic description of the defects. Instead, we assume that the system arranges itself to be on the critical surface associated with MCT. We then investigate the conditions that the defect degrees of freedom must satisfy to be on this critical surface.

The cutoff mechanism of Das and Mazenko [1] will eventually influence the very long time dynamics by generating an exponential decay. We note that there exists a mechanism [22,15] that drives $\gamma(z)$ to a small value so that the observed slowing down is retained. Although, in principle we can include this effect into the coupled equations, we consider here the situation where $\gamma(z)=0$. Another simplification made in this analysis is to neglect the wavenumber dependences of correlation functions. In terms of FNH, one can construct a wavenumber dependent model as shown in Ref. [24]. Since this model is difficult to analyze numerically, we focus here on the wavenumber independent model.

In Sec. II, we present a detailed formulation of FNH of simple fluids including the defect variable. We then use the well-known field-theoretic techniques to calculate the relevent nonlinear contributions to the glass transition. In Sec. [II], the case where the defect autocorrelation is a constant is considered in detail as a first approximation. This will reproduce the results of Ref. [19]. The analysis of the full model which includes the defect dynamics 
is presented in Sec. IV. In Sec. $\mathrm{V}$, we discuss the cutoff mechanism and the temperature dependence of the viscosity in this formulation. In Appendix, we consider the case of a general potential $h(n)$ without assuming the particular double-well form.

\section{FNH WITH THE DEFECT DENSITY VARIABLE}

In this section, we formulate the FNH of compressible fluids in detail including the defect density $n$ as the additional slow variable. Using well-developed field theoretic methods, we calculate nonlinear corrections to the density and defect autocorrelation functions systematically.

\section{A. Generalized Langevin Equations}

Our starting point is the generalized Langevin equation for the set of slow variables, $\psi_{\alpha}=\rho(\mathbf{x}), g_{i}(\mathbf{x}), n(\mathbf{x})$, where $\rho$ is the mass density and $\mathbf{g}$ is the momentum density. Here $\alpha$ labels the type of the field, the position $\mathbf{x}$, and the vector label $i$. Following the standard

procedure described by Ma and Mazenko [23], we have the equation of motion given by

$$
\frac{\partial \psi_{\alpha}}{\partial t}=\bar{V}_{\alpha}[\psi]-\sum_{\beta} \Gamma_{\alpha \beta} \frac{\delta F}{\delta \psi_{\beta}}+\Theta_{\alpha}
$$

where $F$ is the effective Hamiltonian and $\bar{V}_{\alpha}$ is the streaming velocity governing the reversible dynamics and is given by

$$
\bar{V}_{\alpha}[\psi]=\sum_{\beta}\left\{\psi_{\alpha}, \psi_{\beta}\right\} \frac{\delta F}{\delta \psi_{\beta}},
$$

and $\left\{\psi_{\alpha}, \psi_{\beta}\right\}$ is the Poisson bracket among the slow variables. The dissipative matrix $\Gamma_{\alpha \beta}$ and the Gaussian noise $\Theta_{\alpha}$ satisfy

$$
\left\langle\Theta_{\alpha}(t) \Theta_{\beta}\left(t^{\prime}\right)\right\rangle=2 k_{B} T \Gamma_{\alpha \beta} \delta\left(t-t^{\prime}\right) .
$$

The effective Hamiltonian for $\psi_{\alpha}$ is given by

$$
F=F_{K}+F_{u}[\delta \rho]+F_{v}[\delta \rho, n],
$$


where $F_{K}$ is the kinetic energy:

$$
F_{K}=\int d^{3} \mathbf{x} \frac{\mathbf{g}^{2}(\mathbf{x})}{2 \rho(\mathbf{x})}
$$

$F_{u}[\delta \rho]$ is the potential energy for the density fluctuation, $\delta \rho=\rho-\rho_{0}$, where $\rho_{0}$ is the average density, and $F_{v}[\delta \rho, n]$ governs the defect density and its coupling to $\rho$. In general, $F_{u}[\delta \rho]$ can be any local functional of $\delta \rho$ and the spatial derivatives of $\delta \rho$. In particular, one can study the wave-number dependence of the structure factor by including the spatial derivatives [24]. Recently it has been claimed [25] that by including the wave number dependences one can effectively generate the von-Schweidler and the stretching. Analytical treatment in this case, however, is very difficult. In the present case, we consider the simple quadratic form which corresponds to a wave-number independent structure factor,

$$
F_{u}[\delta \rho]=\int d^{3} \mathbf{x} \frac{A}{2}(\delta \rho(\mathbf{x}))^{2}
$$

where $A$ is the flat inverse susceptibility and wavenumbers are restricted to values less than a cutoff $\Lambda . F_{v}[\delta \rho, n]$ in Eq. (2.4) is assumed to be of the form:

$$
F_{v}[\delta \rho, n]=\int d^{3} \mathbf{x}[B \delta \rho(\mathbf{x}) n(\mathbf{x})+h(n(\mathbf{x}))]
$$

where we introduced the simple coupling term through the coupling constant $B$. As discussed in Sec. [1, we construct the potential energy $h(n)$ to be a double well potential [26] with the metastable defect density $\bar{n}$ associated with the higher well. We parametrize $h(n)$ such that $h(n)$ has three extrema at $n=0,(1-\sigma) \bar{n}$ and $\bar{n}$ :

$$
h^{\prime}(n)=\epsilon n(n-(1-\sigma) \bar{n})(n-\bar{n})
$$

or upon integrating,

$$
h(n)=\epsilon \bar{n}^{4}\left[\frac{1}{4}\left(\frac{n}{\bar{n}}\right)^{4}-\frac{1}{3}(2-\sigma)\left(\frac{n}{\bar{n}}\right)^{3}+\frac{1}{2}(1-\sigma)\left(\frac{n}{\bar{n}}\right)^{2}\right]
$$

The parameter $\epsilon$ gives the correct dimension for $h(n)$ and describes the overall scale of the potential energy. For small positive $\sigma$, Eq. (2.9) represents a double well potential with the 
global minimum at $n=0$ and the metastable minimum at $\bar{n}$. We note that for small negative $\sigma, h(n)$ in the above representation is also a double well potential. In fact, $h(n)$ is invariant under $\sigma \rightarrow \sigma^{\prime} \equiv-\sigma /(1-\sigma)$ and $\bar{n} \rightarrow \bar{n}^{\prime} \equiv(1-\sigma) \bar{n}$. Thus, in the following analysis, only the absolute value of $\sigma$ will play a role. We also note that if $\sigma=0$, then $h(n)$ develops an inflexion point at $n=\bar{n}$. It is useful to define two dimensionless parameters $x$ and $y$ which characterize the scale of the coupling energy and the potential energy respectively:

$$
x \equiv \frac{B \rho_{0} \bar{n}}{A \rho_{0}^{2}}, \quad y \equiv \frac{\epsilon \bar{n}^{4}}{A \rho_{0}^{2}} .
$$

The potential and coupling energy are then completely described by $x, y, \sigma$ and $\bar{n}$. We consider the coupling energy term in Eq. (2.7) as a small distortion to the shape of $h(n)$. In particular, $\bar{n}$ is shifted to $n^{*}[\delta \rho]$ determined by

$$
0=\left.\frac{\delta F_{v}}{\delta n}\right|_{n=n^{*}[\delta \rho]}=B \delta \rho+h^{\prime}\left(n^{*}[\delta \rho]\right) .
$$

If we expand in powers of $\delta \rho$,

$$
n^{*}[\delta \rho]=\bar{n}\left[1+a_{1}\left(\frac{\delta \rho}{\rho_{0}}\right)+a_{2}\left(\frac{\delta \rho}{\rho_{0}}\right)^{2}+\cdots\right]
$$

and we can easily calculate the coefficients $a_{1}, a_{2}, \cdots$ using Eq. (2.11):

$$
a_{1}=-\frac{x}{\sigma y}, \quad a_{2}=-\left(\frac{1+\sigma}{\sigma}\right)\left(\frac{x}{\sigma y}\right)^{2}, \quad \ldots .
$$

We will later consider the fluctuation of $n$ around this shifted $n^{*}[\delta \rho]: \quad \delta n=n-n^{*}[\delta \rho]$. Eq. (2.12) indicates that, for small $|\sigma|$, the coupling energy $x$ must be sufficiently small such that

$$
\left|a_{1}\right| \ll 1, \quad\left|a_{2}\right| \ll 1
$$

in order to have a sensible expansion.

Turning to the reversible streaming velocity terms in the Langevin equations, we assume that the Poisson brackets involving $\rho$ and $\mathbf{g}$ are evaluated in the usual way as in Ref. [11]. The new variable $n$ is a scalar quantity as $\rho$, thus we assume that the Poisson brackets for $n$ have the same structure as those for $\rho$. The only nonvanishing elements involving $n$ are 


$$
\begin{aligned}
& \left\{n(\mathbf{x}), g^{i}\left(\mathbf{x}^{\prime}\right)\right\}=-\nabla_{\mathbf{x}}^{i}\left[\delta\left(\mathbf{x}-\mathbf{x}^{\prime}\right) n(\mathbf{x})\right] \\
& \left\{g^{i}(\mathbf{x}), n\left(\mathbf{x}^{\prime}\right)\right\}=\nabla_{\mathbf{x}^{\prime}}^{i}\left[\delta\left(\mathbf{x}-\mathbf{x}^{\prime}\right) n(\mathbf{x})\right]
\end{aligned}
$$

Since there are no Poisson brackets relating $\rho$ to $n$ and $\Gamma_{\rho \beta}=0$, the Langevin equation for $\rho$ is simply the continuity equation,

$$
\frac{\partial \rho}{\partial t}=-\nabla \cdot \mathbf{g}
$$

The streaming velocity for $g^{i}$ then follows from Eq. (2.2):

$$
\begin{aligned}
\bar{V}_{g}^{i} & =\int d^{3} \mathbf{y}\left[\left\{g^{i}(\mathbf{x}), \rho(\mathbf{y})\right\} \frac{\delta F}{\delta \rho(\mathbf{y})}+\left\{g^{i}(\mathbf{x}), n(\mathbf{y})\right\} \frac{\delta F}{\delta n(\mathbf{y})}+\sum_{j}\left\{g^{i}(\mathbf{x}), g^{j}(\mathbf{y})\right\} \frac{\delta F}{\delta g_{j}(\mathbf{y})}\right] \\
& =-\rho(\mathbf{x}) \nabla_{\mathbf{x}}^{i} \frac{\delta F}{\delta \rho(\mathbf{x})}-n(\mathbf{x}) \nabla_{\mathbf{x}}^{i} \frac{\delta F}{\delta n(\mathbf{x})}-\sum_{j} \nabla_{\mathbf{x}}^{j}\left(\frac{g_{i} g_{j}}{\rho}(\mathbf{x})\right)
\end{aligned}
$$

Using the explicit form of the effective Hamiltonian, Eqs. (2.5-2.7), $\bar{V}_{g}^{i}$ can be expressed as a functional of $\delta \rho, g^{i}$ and $n$. In terms of the fluctuation, $\delta n=n-n^{*}[\delta \rho]$, we have, using Eq. (2.12),

$$
\bar{V}_{g}^{i}=-\sum_{j} \nabla_{j}\left(\sigma_{i j}^{u}+\sigma_{i j}^{v}\right)-\sum_{j} \nabla_{j}\left(\frac{g_{i} g_{j}}{\rho}\right),
$$

where

$$
\sigma_{i j}^{u} \equiv \delta_{i j}\left\{c_{0}^{2}(\delta \rho)+\frac{1}{2} \widetilde{A}(\delta \rho)^{2}\right\}
$$

is the usual stress tensor with the definitions,

$$
\begin{aligned}
c_{0}^{2} & \equiv A \rho_{0}\left(1-\frac{x^{2}}{\sigma y}\right) \\
\widetilde{A} & \equiv A\left(1-\frac{x^{2}}{\sigma y}-\frac{2(1+\sigma) x^{3}}{\sigma^{3} y^{2}}\right) .
\end{aligned}
$$

The bare sound speed $c_{0}^{2}$ and the inverse susceptibility $\widetilde{A}$ are modified due to the coupling. But the terms depending on the coupling constant are even smaller than $\left|a_{1}\right|$ and $\left|a_{2}\right|$ by a factor of $|x|$. Thus we may neglect them and regain the usual relations:

$$
c_{0}^{2}=A \rho_{0}, \quad \widetilde{A}=A .
$$


The new $n$-dependent terms in Eq. (2.18) are given by

$$
\sigma_{i j}^{v} \equiv \delta_{i j}\left(A \rho_{0}^{2}\right)\left[(x+\sigma y)\left(\frac{\delta n}{\bar{n}}\right)-2 x\left(\frac{1+\sigma}{\sigma}\right)\left(\frac{\delta \rho}{\rho_{0}}\right)\left(\frac{\delta n}{\bar{n}}\right)+y\left(1+\frac{3 \sigma}{2}\right)\left(\frac{\delta n}{\bar{n}}\right)^{2}\right] .
$$

For our purpose, we need only to keep upto quadratic terms in the fluctuating quantities. The Langevin equation for $g^{i}$ can then be written as

$$
\frac{\partial g^{i}}{\partial t}=-\sum_{j} \nabla_{j}\left(\sigma_{i j}^{u}+\sigma_{i j}^{v}\right)-\sum_{j} \nabla_{j}\left(\frac{g^{i} g^{j}}{\rho}\right)-\sum_{j} L_{i j}\left(\frac{g^{j}}{\rho}\right)+\Theta_{i},
$$

where $L_{i j}(\mathbf{x})=-\eta_{0}\left(\frac{1}{3} \nabla_{i} \nabla_{j}+\delta_{i j} \nabla^{2}\right)-\zeta_{0} \nabla_{i} \nabla_{j}$ with the bare shear and bulk viscosities, $\eta_{0}$ and $\zeta_{0}$, respectively. For later use, we define the bare longitudinal viscosity, $\Gamma_{0} \equiv \zeta_{0}+\frac{4}{3} \eta_{0}$. In Eq. (2.24), the Gaussian noise $\Theta_{i}$ satisfies

$$
\left\langle\Theta_{i}(\mathbf{x}, t) \Theta_{j}\left(\mathbf{x}^{\prime}, t^{\prime}\right)\right\rangle=2 k_{B} T L_{i j}(\mathbf{x}) \delta\left(\mathbf{x}-\mathbf{x}^{\prime}\right) \delta\left(t-t^{\prime}\right) .
$$

The streaming velocity for $n$ is given by

$$
\bar{V}_{n}=\int d^{3} \mathbf{x}^{\prime} \sum_{i}\left\{n(\mathbf{x}), g^{i}\left(\mathbf{x}^{\prime}\right)\right\} \frac{\delta F}{\delta g^{i}\left(\mathbf{x}^{\prime}\right)}=-\sum_{i} \nabla_{\mathbf{x}}^{i}\left(n(\mathbf{x}) \frac{g^{i}(\mathbf{x})}{\rho(\mathbf{x})}\right) .
$$

Assuming $n$ is a diffusive variable, we must choose $\Gamma_{n n}\left(\mathbf{x}-\mathbf{x}^{\prime}\right) \equiv-\Gamma_{v} \nabla_{\mathbf{x}}^{2} \delta\left(\mathbf{x}-\mathbf{x}^{\prime}\right)$ and the associated noise $\Xi$ satisfies

$$
<\Xi(\mathbf{x}, t) \Xi\left(\mathbf{x}^{\prime}, t^{\prime}\right)>=-2 k_{B} T \Gamma_{v} \nabla_{x}^{2} \delta\left(\mathbf{x}-\mathbf{x}^{\prime}\right) \delta\left(t-t^{\prime}\right)
$$

We have the Langevin equation for $n$,

$$
\frac{\partial n}{\partial t}=-\sum_{i} \nabla_{i}\left(n \frac{g^{i}}{\rho}\right)+\Gamma_{v} \nabla^{2} \frac{\delta F}{\delta n}+\Xi .
$$

As in the case of $g^{i}$, we expand $n$ around the metastable state $n^{*}[\delta \rho]$ and write the equation in terms of the fluctuation $\delta n$. Using Eq. (2.12) and keeping up to quadratic terms in fluctuating variables, we have

$$
\begin{aligned}
\frac{\partial}{\partial t}(\delta n)= & -\bar{n}\left(1+\frac{x}{\sigma y}\right) \nabla \cdot\left(\frac{\mathbf{g}}{\rho}\right)-\nabla \cdot\left[(\delta n) \frac{\mathbf{g}}{\rho}\right] \\
& +\bar{n} \widetilde{\Gamma}_{v} \nabla^{2}\left[\sigma y\left(\frac{\delta n}{\bar{n}}\right)-2 x\left(\frac{1+\sigma}{\sigma}\right)\left(\frac{\delta \rho}{\rho_{0}}\right)\left(\frac{\delta n}{\bar{n}}\right)+y(1+\sigma)\left(\frac{\delta n}{\bar{n}}\right)^{2}\right]+\Xi,
\end{aligned}
$$

with the rescaled diffusive coefficient

$$
\widetilde{\Gamma}_{v} \equiv \frac{A \rho_{0}^{2}}{\bar{n}^{2}} \Gamma_{v}
$$




\section{B. Field-theoretic Formulation}

The Langevin equations, Eqs. 2.16), (2.24) and (2.29) can be put into a field theoretical form following standard Martin-Siggia-Rose (MSR) procedures [12,13. It essentially amounts to introducing the hatted variable $\widehat{\psi}_{\alpha}$ for each field $\psi_{\alpha}$ to enforce the equations of motion and integrating out the Gaussian noises to get the quadratic form in $\widehat{\psi}_{\alpha}$. We introduce the local velocity field $\mathbf{V}$, where $\mathbf{g}=\rho \mathbf{V}$, to eliminate the $1 / \rho$ nonlinearities 11 in Eqs. (2.24) and (2.29). This relation is then enforced by inserting the identity

$$
\begin{aligned}
1 & =\int \mathcal{D} \mathbf{V} \delta\left(\frac{\mathbf{g}}{\rho}-\mathbf{V}\right)=\|\rho\| \int \mathcal{D} \mathbf{V} \delta(\mathbf{g}-\rho \mathbf{V}) \\
& =\|\rho\| \int \mathcal{D} \widehat{\mathbf{V}} \mathcal{D} \mathbf{V} \exp \left(i \int d 1 \widehat{\mathbf{V}}(1) \cdot(\mathbf{g}(1)-\rho(1) \mathbf{V}(1))\right)
\end{aligned}
$$

into the functional integral form, where $\|\rho\|$ is the functional Jacobian between two delta functionals, and $d 1 \equiv d^{3} \mathbf{x}_{1} d t_{1}, \mathbf{g}(1) \equiv \mathbf{g}\left(\mathbf{x}_{1}, t_{1}\right)$, and so on. As shown in Ref. [27], the Jacobian $\|\rho\|$ has no dynamical effect, so that it can be neglected throughout the analysis. The generating functional is then given in terms of a functional integral over $\Psi_{\alpha}, \widehat{\Psi}_{\alpha}$, where $\Psi_{\alpha}=\left\{\delta \rho, g_{i}, \delta n, V_{i}\right\}$. Without source terms, it is given by

$$
Z=\int \mathcal{D} \Psi \mathcal{D} \widehat{\Psi} \exp (-S[\Psi, \widehat{\Psi}])
$$

where the action $S[\Psi, \widehat{\Psi}]$ is given by

$$
\begin{aligned}
S[\Psi, \widehat{\Psi}]=\int d 1[ & \sum_{i j} \widehat{g}_{i} \beta^{-1} L_{i j}(1) \widehat{g}_{j}-\widehat{\delta n} \beta^{-1} \Gamma_{v} \nabla^{2} \widehat{\delta n} \\
& +i \widehat{\delta \rho}\left[\frac{\partial \rho}{\partial t}+\nabla \cdot \mathbf{g}\right]+i \sum_{i} \widehat{V}_{i}\left[g_{i}-\rho V_{i}\right] \\
& +i \sum_{i} \widehat{g}_{i}\left[\frac{\partial g_{i}}{\partial t}+\sum_{j} \nabla^{j}\left(\sigma_{i j}^{u}+\sigma_{i j}^{v}\right)+\sum_{j}\left[\nabla^{j}\left(\rho V_{i} V_{j}\right)+L_{i j}(1)\left(V_{j}\right)\right]\right] \\
& +i \widehat{\delta n}\left[\frac{\partial \delta n}{\partial t}+\bar{n}\left(1+\frac{x}{\sigma y}\right) \nabla \cdot \mathbf{V}+\nabla \cdot[(\delta n) \mathbf{V}]\right. \\
& \left.\left.\quad-\bar{n} \widetilde{\Gamma}_{v} \nabla^{2}\left[\sigma y\left(\frac{\delta n}{\bar{n}}\right)-2 x\left(\frac{1+\sigma}{\sigma}\right)\left(\frac{\delta \rho}{\rho_{0}}\right)\left(\frac{\delta n}{\bar{n}}\right)+(1+\sigma) y\left(\frac{\delta n}{\bar{n}}\right)^{2}\right]\right]\right]
\end{aligned}
$$

where $\beta^{-1}=k_{B} T$. As a result of Eq. (2.31), we have a polynomial action in $\Psi_{\alpha}$ and $\widehat{\Psi}_{\alpha}$, to which we can apply the perturbation theory expansion in a standard way. The nonlinear 
corrections coming from the cubic terms [28] in the action generate the one-loop self-energies $\Sigma_{\alpha \beta}$ which modify the zeroth order inverse propagators $\left[G_{\alpha \beta}^{0}\right]^{-1}$ through Dyson's equation:

$$
G_{\alpha \beta}^{-1}=\left[G_{\alpha \beta}^{0}\right]^{-1}-\Sigma_{\alpha \beta}
$$

\section{Linearized Theory}

From the quadratic terms in the action, Eq. (2.33), we can easily read off the elements

of the zeroth order inverse propagator, $\left[G_{\alpha \beta}^{0}\right]^{-1}$. By inverting this matrix, we get various correlation and linear response functions. For later use, we list here a few of these functions. In this paper, we will be concerned only with the longitudinal parts of those functions and use hereafter the notation $\rho, n$ instead of $\delta \rho, \delta n$ respectively for convenience. In the Fourier-transformed space, we have

$$
\begin{aligned}
& G_{n \hat{n}}^{0}(\mathbf{q}, \omega)=-G_{\widehat{n} n}^{0 *}(\mathbf{q}, \omega)=\frac{D_{0}}{W_{0}}, \\
& G_{\rho \hat{\rho}}^{0}(\mathbf{q}, \omega)=\frac{1}{W_{0}}\left[\left(\omega+i \sigma y \widetilde{\Gamma}_{v} q^{2}\right)\left(\rho_{0} \omega+i \Gamma_{0} q^{2}\right)-A \rho_{0}^{2} \frac{(x+\sigma y)^{2}}{\sigma y} q^{2}\right],
\end{aligned}
$$

where

$$
\begin{aligned}
& D_{0}(\mathbf{q}, \omega)=\rho_{0}\left(\omega^{2}-q^{2} c_{0}^{2}\right)+i \omega q^{2} \Gamma_{0} \\
& W_{0}(\mathbf{q}, \omega)=\left(\omega+i \sigma y \widetilde{\Gamma}_{v} q^{2}\right) D_{0}-\frac{(x+\sigma y)^{2}}{\sigma y} \omega q^{2} .
\end{aligned}
$$

The density-density and defect-defect correlation functions are given respectively by

$$
\begin{aligned}
& G_{n n}^{0}(\mathbf{q}, \omega)=\bar{n}^{2} \frac{2 \beta^{-1} q^{2}}{\left|W_{0}\right|^{2}}\left[\left(\frac{x+\sigma y}{\sigma y}\right)^{2} \Gamma_{0} \omega^{2} q^{2}+\frac{\widetilde{\Gamma}_{v}}{A \rho_{0}^{2}}\left|D_{0}\right|^{2}\right], \\
& G_{\rho \rho}^{0}(\mathbf{q}, \omega)=\rho_{0}^{2} \frac{2 \beta^{-1} q^{4}}{\left|W_{0}\right|^{2}}\left[\Gamma_{0}\left|\omega+i \sigma y \widetilde{\Gamma}_{v} q^{2}\right|^{2}+A \rho_{0}^{2}(x+\sigma y)^{2} \widetilde{\Gamma}_{v} q^{2}\right] .
\end{aligned}
$$

Other correlation functions involving $n$ are given by

$$
G_{\rho n}^{0}(\mathbf{q}, \omega)=\rho_{0} \bar{n} \frac{2 \beta^{-1} q^{4}}{\left|W_{0}\right|^{2}}\left(\frac{x+\sigma y}{\sigma y}\right)\left[\omega^{2} \Gamma_{0}+\sigma y \widetilde{\Gamma}_{v} \rho_{0}\left(\omega^{2}-q^{2} c_{0}^{2}\right)\right]
$$

and 


$$
G_{g n}^{0}(\mathbf{q}, \omega)=\rho_{0} G_{V n}^{0}(\mathbf{q}, \omega)=\left(\frac{\omega}{q}\right) G_{\rho n}^{0}(\mathbf{q}, \omega)
$$

We note that the zeroth order response and correlation functions satisfy the following fluctuation-dissipation theorem:

$$
\begin{aligned}
& G_{\alpha n}^{0}(\mathbf{q}, \omega)=-\frac{1}{\sigma y}\left(\frac{\bar{n}}{\rho_{0}}\right)^{2} \frac{2 \beta^{-1}}{A} \operatorname{Im} G_{\alpha \widehat{n}}^{0}(\mathbf{q}, \omega), \\
& G_{\rho \rho}^{0}(\mathbf{q}, \omega)=-\frac{2 \beta^{-1}}{A} \operatorname{Im} G_{\rho \hat{\rho}}^{0}(\mathbf{q}, \omega),
\end{aligned}
$$

where $\alpha=\left\{\rho, g_{i}, V_{i}, n\right\}$.

From Eqs. (2.35-2.38), we find that if we can neglect the factor

$$
\left|\frac{(x+\sigma y)^{2}}{\sigma y}\right|=\left|\sigma y\left(1-a_{1}\right)^{2}\right| \ll 1,
$$

then we have

$$
G_{n \hat{n}}^{0}(\mathbf{q}, \omega) \simeq \frac{1}{\omega+i \gamma_{v}}
$$

and

$$
G_{\rho \widehat{\rho}}^{0}(\mathbf{q}, \omega) \simeq \frac{\rho_{0} \omega+i \Gamma_{0} q^{2}}{D_{0}(\mathbf{q}, \omega)}
$$

where $\gamma_{v} \equiv \sigma y \widetilde{\Gamma}_{v} q^{2}$. The assumption in Eq. (2.44) is equivalent to saying that we are considering the small $|\sigma|$ limit, which will be justified self-consistently later when we consider the conditions for the slowing down. Eq. (2.46) represents a standard form for the density auto-correlation function. The defect auto-correlation function in Eq. (2.45) has a very slow diffusive mode, $\omega=-i \gamma_{v}$, since it is assumed that $\gamma_{v} \ll 1$. This signals a separation of time scales between the density and the defect variables as noted in Sec. [1.

We can achieve a further simplification by looking at the following situation. As one approaches the glass transition, we expect that, since the viscosity is getting extremely large, one eventually reaches a point where

$$
\frac{q^{2} \Gamma_{0}}{\rho_{0}} \gg \omega
$$


Then, from Eqs. (2.38,2.44), the denominator $W_{0}$ in Eqs. (2.35) and (2.36) reduces to

$$
\begin{aligned}
W_{0} & \simeq i q^{2} \Gamma_{0}\left\{\omega^{2}+i\left(\gamma_{v}+\gamma_{u}\right) \omega-\gamma_{v} \gamma_{u}\right\} \\
& =i q^{2} \Gamma_{0}\left(\omega+i \gamma_{v}\right)\left(\omega+i \gamma_{u}\right)
\end{aligned}
$$

where $\gamma_{u} \equiv A \rho_{0}^{2} / \Gamma_{0}$. It follows then from Eqs. (2.39-2.41) that

$$
\begin{aligned}
G_{n n}^{0}(\mathbf{q}, \omega) & \simeq \frac{1}{\sigma y}\left(\frac{\bar{n}}{\rho_{0}}\right)^{2} \frac{2 \beta^{-1}}{A} \frac{\gamma_{v}}{\omega^{2}+\gamma_{v}^{2}}, \\
G_{\rho \rho}^{0}(\mathbf{q}, \omega) & \simeq \frac{2 \beta^{-1}}{A} \frac{\gamma_{u}}{\omega^{2}+\gamma_{u}^{2}}, \\
G_{\rho n}^{0}(\mathbf{q}, \omega) & \simeq \frac{\bar{n}}{\rho_{0}}\left(\frac{x+\sigma y}{\sigma y}\right) \frac{2 \beta^{-1}}{A}\left(-\frac{\gamma_{v}}{\omega^{2}+\gamma_{v}^{2}}+\frac{\gamma_{u}}{\omega^{2}+\gamma_{u}^{2}}\right) \\
& \simeq-\frac{\rho_{0}}{\bar{n}}(x+\sigma y) G_{n n}^{0}(\mathbf{q}, \omega)+\frac{\bar{n}}{\rho_{0}}\left(\frac{x+\sigma y}{\sigma y}\right) G_{\rho \rho}^{0}(\mathbf{q}, \omega) .
\end{aligned}
$$

Therefore, in this approximation, we have the density-defect correlation function as a linear combination of the density and the defect auto-correlation functions.

\section{Nonlinear Corrections}

Nonlinear corrections to the zeroth order response and correlation functions in the previous section are represented in terms of the self-energies through Eq. (2.34). For example, Eqs. (2.36,2.35) are modified to yield very complicated expressions:

$$
\begin{aligned}
G_{n \widehat{n}}(\mathbf{q}, \omega)=\frac{D(\mathbf{q}, \omega)}{W(\mathbf{q}, \omega)} & \\
G_{\rho \widehat{\rho}}(\mathbf{q}, \omega)=\frac{1}{W(\mathbf{q}, \omega)}[ & {\left[\omega+i \gamma_{v}^{\prime}(\mathbf{q}, \omega)\right]\left[\rho(\mathbf{q}, \omega) \omega+i \Gamma(\mathbf{q}, \omega) q^{2}\right] } \\
& \left.-q \bar{n}^{\prime}(\mathbf{q}, \omega)\left[q u^{\prime}(\mathbf{q}, \omega)+i \omega \Sigma_{\widehat{V} n}(\mathbf{q}, \omega)\right]\right],
\end{aligned}
$$

where

$$
\begin{aligned}
W(\mathbf{q}, \omega) \equiv & {\left[\omega+i \gamma_{v}^{\prime}(\mathbf{q}, \omega)\right] D(\mathbf{q}, \omega) } \\
& -q^{2} u^{\prime}(\mathbf{q}, \omega)\left[\bar{n}^{\prime}(\mathbf{q}, \omega)\left[\omega+i q \Sigma_{\widehat{V} \rho}(\mathbf{q}, \omega)\right]+\rho(\mathbf{q}, \omega) \Sigma_{\widehat{n} \rho}(\mathbf{q}, \omega)\right] \\
& \left.-i q \Sigma_{\widehat{V} n}(\mathbf{q}, \omega)\left[\bar{n}^{\prime}(\mathbf{q}, \omega)\left[\omega^{2}-q^{2} c^{2}(\mathbf{q}, \omega)\right]-i \Gamma(\mathbf{q}, \omega) q^{2} \Sigma_{\widehat{n} \rho}(\mathbf{q}, \omega)\right]\right]
\end{aligned}
$$


and

$$
D(\mathbf{q}, \omega) \equiv \rho(\mathbf{q}, \omega)\left[\omega^{2}-q^{2} c^{2}(\mathbf{q}, \omega)\right]+i \Gamma(\mathbf{q}, \omega) q^{2}\left[\omega+i q \Sigma_{\widehat{V} \rho}(\mathbf{q}, \omega)\right]
$$

with the renormalization of parameters given by

$$
\begin{aligned}
& \rho(\mathbf{q}, \omega)=\rho_{0}-i \Sigma_{\widehat{V} V}^{L}(\mathbf{q}, \omega) \\
& q c^{2}(\mathbf{q}, \omega)=q c_{0}^{2}+\Sigma_{\widehat{g} \rho}(\mathbf{q}, \omega) \\
& q^{2} \Gamma(\mathbf{q}, \omega)=q^{2} \Gamma_{0}+i \Sigma_{\widehat{g} V}^{L}(\mathbf{q}, \omega) \\
& \gamma_{v}^{\prime}(\mathbf{q}, \omega)=\gamma_{v}+i \Sigma_{\widehat{n} n}(\mathbf{q}, \omega) \\
& q \bar{n}^{\prime}(\mathbf{q}, \omega)=q\left(1-a_{1}\right) \bar{n}+\Sigma_{\widehat{n} V}(\mathbf{q}, \omega) \\
& q u^{\prime}(\mathbf{q}, \omega)=q\left(B \rho_{0}+\sigma \epsilon \bar{n}^{3}\right)+\Sigma_{\widehat{g} n}(\mathbf{q}, \omega) \\
& =(x+\sigma y) \frac{A \rho_{0}^{2}}{\bar{n}}+\Sigma_{\widehat{g} n}(\mathbf{q}, \omega) .
\end{aligned}
$$

In the above equation $L$ denotes the longitudinal part of self-energy. For example, $\Sigma_{\widehat{V}_{i} V_{j}}(\mathbf{q}, \omega)$ is decomposed into the longitudinal and transverse parts:

$$
\Sigma_{\widehat{V}_{i} V_{j}}(\mathbf{q}, \omega)=\frac{q_{i} q_{j}}{q^{2}} \Sigma_{\widehat{V} V}^{L}(\mathbf{q}, \omega)+\left(\delta_{i j}-\frac{q_{i} q_{j}}{q^{2}}\right) \Sigma_{\widehat{V} V}^{T}(\mathbf{q}, \omega) .
$$

As shown by Das and Mazenko [11], the self-energy $\Sigma_{\widehat{V} \rho}(\mathbf{q}, \omega)$ is responsible for the cutoff mechanism. In addition to that, we have self-energies $\Sigma_{\widehat{V} n}(\mathbf{q}, \omega)$ and $\Sigma_{\widehat{n} \rho}(\mathbf{q}, \omega)$ in this case. As will be discussed in Sec. $\nabla$, all three self-energies are related to the cutoff mechanism. Although the final stage of relaxation is governed by this cutoff effect, we neglect it in the present analysis.

The separation of time scales observed in the zeroth order correlation functions remains valid at high orders if the renormalized version of Eq. (2.44) holds, i.e.,

$$
\left|\frac{u^{\prime}(\mathbf{q}, \omega) \bar{n}^{\prime}(\mathbf{q}, \omega)}{\rho(\mathbf{q}, \omega) c^{2}(\mathbf{q}, \omega)}\right| \ll 1 .
$$

In this case, Eqs. (2.52,2.53) give us

$$
\begin{aligned}
& G_{n \widehat{n}}(\mathbf{q}, \omega) \simeq \frac{1}{\omega+i \gamma_{v}^{\prime}(\mathbf{q}, \omega)} \\
& G_{\rho \widehat{\rho}}(\mathbf{q}, \omega) \simeq \frac{\rho(\mathbf{q}, \omega) \omega+i \Gamma(\mathbf{q}, \omega) q^{2}}{\rho(\mathbf{q}, \omega)\left[\omega^{2}-q^{2} c^{2}(\mathbf{q}, \omega)\right]+i \Gamma(\mathbf{q}, \omega) \omega q^{2}} .
\end{aligned}
$$


Eqs. (2.59) and (2.60) are the fundamental equations for the dynamics of density and defect fluctuations without the cutoff effect. In the following, we calculate explicitly nonlinear contributions to the renormalized viscosity $\Gamma(\mathbf{q}, \omega)$ and the renormalized diffusion coefficient $\gamma_{v}^{\prime}(\mathbf{q}, \omega)$, which will be expressed back in terms of $G_{n \widehat{n}}(\mathbf{q}, \omega)$ and $G_{\rho \widehat{\rho}}(\mathbf{q}, \omega)$.

\section{E. One-Loop Evaluation of $\Gamma(\mathbf{q}, \omega)$ and $\gamma_{v}^{\prime}(\mathbf{q}, \omega)$}

The density feedback mechanism of MCT is realized by calculating the nonlinear corrections to the bare viscosity in the density correlation function of the form, Eq. (2.60). In the present case, however, we have two coupled equations, (2.59) and (2.60). Thus we must find both kinetic coefficients, $\Gamma(\mathbf{q}, \omega)$ and $\gamma_{v}^{\prime}(\mathbf{q}, \omega)$ to complete the equations. In this section, we calculate one-loop nonlinear contributions to those quantities.

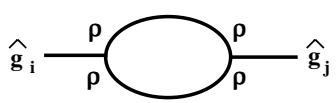

(a)

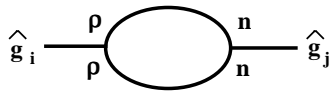

(c)

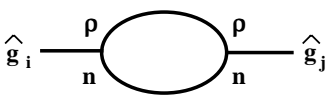

(e)

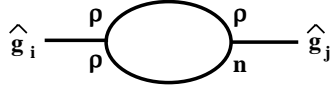

(b)

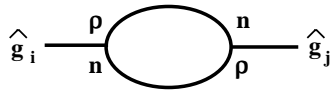

(d)

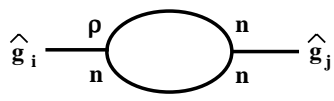

(f)

One-loop diagrams contributing to $\Sigma_{\widehat{g} \widehat{g}}(\mathbf{q}, \omega)$

FIG. 2

As noted in Ref. [11], the renormalized longitudinal viscosity in Eq. (2.560) can also be represented in the hydrodynamic limit as

$$
\Gamma(\mathbf{q}, \omega)=\Gamma_{0}-\frac{\beta}{2 q^{2}} \Sigma_{\widetilde{g} g}^{L}(\mathbf{q}, \omega) .
$$

The relevant one-loop diagrams contributing to this self-energy are listed in Fig. 2. As discussed in Ref. [11], the diagrams coming from the convective vertex ( $\widehat{\rho} \rho V V$ vertex) in 
the action Eq. (2.33) just renormalizes the bare viscosity and are irrelevant to the density feedback mechanism.

We note that the approximation given by Eq. (2.51) generates two kinds of terms that are proportional to $G_{n n} G_{\rho \rho}$ and $G_{\rho \rho} G_{\rho \rho}$, respectively, in the expression for $\Gamma(\mathbf{q}, \omega)$. In fact, one can easily see that the $G_{n n} G_{\rho \rho}$ type terms come from diagrams (b),(c),(d),(e) and (f), and the $G_{\rho \rho} G_{\rho \rho}$ type terms from diagrams (a),(b),(c) and (d) in Fig. 2. Thus we have

$$
\begin{aligned}
\Gamma(\mathbf{q}, \omega)=\Gamma_{0}+\int_{0}^{\infty} d t e^{i \omega t} \int \frac{d^{3} \mathbf{k}}{(2 \pi)^{3}} \\
\quad\left[\left[V^{(1)}(\mathbf{q}, \mathbf{k}) G_{n n}(\mathbf{k}, t)+V^{(2)}(\mathbf{q}, \mathbf{k}) G_{\rho \rho}(\mathbf{k}, t)\right] G_{\rho \rho}(\mathbf{q}-\mathbf{k}, t)\right],
\end{aligned}
$$

where $V^{(1)}$ and $V^{(2)}$ are appropriate vertices to be evaluated. In principle, the wave number dependence of the vertices can be considered [24], for example, by using the spatial derivatives of the density fluctuations in the effective Hamiltonian, Eq. (2.6). This involves, however, very complicated wavenumber integrals. In this analysis, we consider the wavenumber independent case. A closely related approximation to this is that the correlation functions can be factorized into wavenumber and time dependent parts [29]. We assume here that $G_{n n}(\mathbf{q}, t)=T(q) \psi(t), G_{\rho \rho}(\mathbf{q}, t)=S(q) \phi(t), T(q)$ and $S(q)$ are the flat structure factors given by

$$
T(q)=\left(\frac{\bar{n}}{\rho_{0}}\right)^{2} \frac{1}{\sigma y} \frac{\beta^{-1}}{A}, \quad S(q)=\frac{\beta^{-1}}{A},
$$

for $q<\Lambda$ and $T(q)=S(q)=0$ for $q>\Lambda$, where $\Lambda$ is the large momentum cutoff. Integrating over wavenumbers for the one-loop diagrams in Fig. 2 with the help of $\Lambda$, we obtain

$$
\Gamma(\omega)=\Gamma_{0}+k_{B} T \frac{\Lambda^{3}}{6 \pi^{2}} \int_{0}^{\infty} d t e^{i \omega t}\left[d_{1} \psi(t) \phi(t)+d_{2} \phi^{2}(t)\right]
$$

where the coefficients $d_{1}$ and $d_{2}$ are functions of $x, y$ and $\sigma$. We find, through detailed calculation of the diagrams that

$$
\begin{aligned}
d_{1}(x, y, \sigma)= & -4 x\left(\frac{1+\sigma}{\sigma}\right)^{2}\left(2+\frac{x}{\sigma y}\right) \\
& -4\left[y(2+3 \sigma)+2 x^{2}\left(\frac{1+\sigma}{\sigma}\right)^{2}\right]\left(1+\frac{x}{\sigma y}\right)^{2},
\end{aligned}
$$




$$
\begin{aligned}
d_{2}(x, y, \sigma)= & 1-4 x\left(\frac{1+\sigma}{\sigma}\right)\left(1+\frac{x}{\sigma y}\right) \\
& +2\left[y(2+3 \sigma)+2 x^{2}\left(\frac{1+\sigma}{\sigma}\right)^{2}\right]\left(1+\frac{x}{\sigma y}\right)^{2} .
\end{aligned}
$$

For later use, we define a dimensionless parameter containing an explicit factor of the temperature,

$$
\xi \equiv \frac{k_{B} T}{A \rho_{0}^{2}} \frac{\Lambda^{3}}{6 \pi^{2}}
$$

The renormalization of $\gamma_{v}$ is given by Eq. (2.56d). As in Eq. (2.61), it is equivalent in the hydrodynamic limit to

$$
\gamma_{v}^{\prime}(\mathbf{q}, \omega)=\gamma_{v}-\frac{1}{2} \sigma y\left(\frac{\rho_{0}}{\bar{n}}\right)^{2} \frac{A}{\beta^{-1}} \Sigma_{\widehat{n} \widehat{n}}(\mathbf{q}, \omega)
$$

Among the nonlinear terms in Eq. (2.33) that contribute, at the one-loop order, to the self-energy $\Sigma_{\widehat{n} \widehat{n}}(\mathbf{q}, \omega)$, those of $\widehat{n} \rho n$ and $\widehat{n} n n$ types contain an explicit factor of $\Gamma_{v}$ which is assumed to be very small. The other one-loop diagrams that contribute to $\Sigma_{\widehat{n} \widehat{n}}(\mathbf{q}, \omega)$, and do not have the explicit $\Gamma_{v}$ factor are listed in Fig. 3.

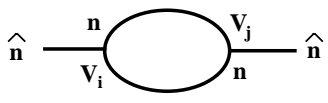

(a)

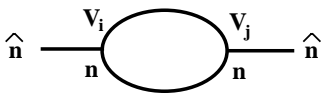

(b)

\section{One-loop diagrams contributing to $\Sigma_{\widehat{n} \widehat{n}}(\mathbf{q}, \omega)$}

FIG. 3

Evaluating the diagram (a) using Eqs. (2.42) and (2.51), we find that this contribution is directly proportional to

$$
\left|\frac{(x+\sigma y)^{2}}{\sigma y}\right| \ll 1
$$

which is our self-consistent assumption, Eq. (2.44). The evaluation of the second diagram (b) yields

$$
\frac{1}{2} \sigma y\left(\frac{\rho_{0}}{\bar{n}}\right)^{2} \frac{A}{\beta^{-1}} \Sigma_{\widehat{n n}}^{(b)}(\mathbf{q}, \omega)=\left(\frac{q}{\Lambda}\right)^{2} \xi \int_{0}^{\infty} d t e^{i \omega t} \psi(t) \frac{d^{2} \phi(t)}{d t^{2}}+I_{T},
$$


where we have used Eq. (2.42). The second term $I_{T}$ in Eq. (2.69) is the integral containing the transverse part of the velocity auto-correlation function, $G_{V V}^{T}(\mathbf{q}, \omega)$. We note that in the framework of the flat structure factor, Eq. (2.63), there is no coupling between the density feedback mechanism and the transverse viscosity. Thus, we assume that this term is insensitive to any dramatic slowing down. We obtain finally that

$$
\gamma_{v}^{\prime}(\mathbf{q}, \omega)=\gamma_{v}-\left(\frac{q}{\Lambda}\right)^{2} \xi \int_{0}^{\infty} d t e^{i \omega t} \psi(t) \ddot{\phi}(t)
$$

Eqs. (2.59) and (2.60) together with Eqs. (2.64) and (2.70) complete the specification of our model.

\section{THE MODEL WITH A CONSTANT DEFECT AUTO-CORRELATION FUNCTION}

Metastability plays an important role in this model, since defects spend considerable amount of time trapped within the well. During this period, we may assume that the defect auto-correlation function can be regarded as a constant:

$$
G_{n n}(\mathbf{q}, \omega)=2 \pi\left(\frac{\bar{n}}{\rho_{0}}\right)^{2} \frac{1}{\sigma y} \frac{\beta^{-1}}{A} \delta(\omega), \quad \text { or } \quad \psi(t)=1
$$

This approxiamtion corresponds to the limit where one takes the bare value of $\gamma_{v}$ to zero. In this case, we note that the nonlinear correction to $\gamma_{v}$ also vanishes in the long time limit, $\omega \rightarrow 0$, since the integrand in Eq. (2.70) is a total derivative when $\psi(t)=1$. Thus the self-consistency of the approximation is maintained.

The coupled equations $(2.59)$ and $(2.60)$ then reduce to a form dependent only on $\phi(t)$ and we can make contact with the standard treatment of MCT [1]. Indeed Eqs. (2.60) and (2.64) with (2.65) are just $N=2$ case of the standard MCT equations, (1.1) and (1.2), where only the linear $\left(c_{1}\right)$ and quadratic $\left(c_{2}\right)$ terms are considered. We can identify $\Omega_{0}^{2}=q^{2} c^{2}$, $d_{0}=q^{2} \Gamma_{0} / \rho$, and

$$
c_{1}=\xi d_{1}(x, y, \sigma), \quad c_{2}=\xi d_{2}(x, y, \sigma)
$$


It is important to note that the MCT coefficients $c_{1}$ and $c_{2}$ are expressed in terms of the temperature and the parameters describing the metastable potential and the coupling between the density and the defect variables. We now assume that the system organizes itself to be on the critical surface of MCT, which is described below. This will give relations among the metastability parameters.

The density feedback mechanism associated with the representation of the type Eq. (1.1) with $N=2$ was first studied by Götze [10]. According to Ref. [10], there exists a critical line in the $\left(c_{1}, c_{2}\right)$ space separating ergodic and nonergodic regions, where the nonergodic phase is characterized by the existence of the limit $f=\lim _{t \rightarrow \infty} \phi(t), \quad f>0$. To study the critical condition in terms of the parameters $x, y, \sigma$ and $\xi$, we follow a more general discussion on such model given by Kim and Mazenko [15]. According to Ref. [15], the glass transition can be described by the following three parameters:

$$
\begin{aligned}
& \sigma_{0}=(1-f) V(f) \\
& \sigma_{1}=(1-f)^{2} V^{\prime}(f) \\
& \lambda=\frac{1}{2}(1-f)^{3} H^{\prime \prime}(f),
\end{aligned}
$$

where $V(f)=H(f)-f /(1-f)$ and $H(f)=\sum_{i=1}^{N} c_{i} f^{i}$ for a general model containing higher order terms in the mode coupling integral. In Eq. (3.5), the parameter $\lambda$ is directly related to the exponents of the sequence of time relaxations [10]:

$$
\frac{\Gamma^{2}(1-a)}{\Gamma(1-2 a)}=\lambda=\frac{\Gamma^{2}(1+b)}{\Gamma(1+2 b)} .
$$

The ideal glass transition is approached when both $\sigma_{0}$ and $\sigma_{1}$ are getting small. Let us consider the situation where $\sigma_{1}=0$ and the transition is approached by taking $\sigma_{0} \rightarrow 0$. Solving Eqs. (3.3-3.5) for $c_{1}$ and $c_{2}$, we have

$$
\begin{aligned}
& c_{1}=\frac{2 \lambda-1}{\lambda^{2}}+\frac{4 \sigma_{0}}{\lambda(1-\lambda)}+\mathcal{O}\left(\sigma_{0}^{2}\right) \\
& c_{2}=\frac{1}{\lambda^{2}}-\frac{3 \sigma_{0}}{\lambda(1-\lambda)^{2}}+\mathcal{O}\left(\sigma_{0}^{2}\right) .
\end{aligned}
$$

In terms of the parameters, $x, y, \sigma$ and $\xi$, the critical condition is given by setting $\sigma_{0}=0$ : 


$$
\begin{aligned}
& d_{1}(x, y, \sigma)=\frac{2 \lambda-1}{\xi \lambda^{2}}, \\
& d_{2}(x, y, \sigma)=\frac{1}{\xi \lambda^{2}}, \quad \frac{1}{2}<\lambda<1 .
\end{aligned}
$$

We note that we are mainly concerned with the region where $|x| \ll 1$ in order to be consistent with the fact that we considered the coupling term as a small perturbation shifting the metastable state as in Eqs. (2.12) and (2.14). We also require the solution be consistent with the assumption, Eq. (2.44). We find that nontrivial solutions to Eq. (3.8) satisfying Eqs. (2.14) and (2.44) exist only if we take $\sigma \rightarrow 0$ simultaneously with $x \rightarrow 0$, while holding

$$
\frac{x}{\sigma^{2}} \rightarrow C
$$

for some constant $C$. Let us briefly discuss the physical meaning of Eq. (3.9). The small $|\sigma|$ limit is a sufficient condition for a separation of the time scales between the defect and the density variables as seen from Eq. (2.44). This indicates that the metastable wells become very shallow. If the coupling energy represented by $|x|$ is stronger than the above limit, i.e. $|x| \sim \mathcal{O}(|\sigma|)$, then the metastable state given by Eq. (2.12) does not exist, since the condition Eq. (2.14) is violated. Thus, a strong coupling energy destroys the metastability of defects. On the other hand, if $|x| \sim \mathcal{O}\left(|\sigma|^{3}\right)$, we have from Eq. (2.65),

$$
d_{1}=-8 y, \quad d_{2}=1+4 y
$$

Since $y$ is always positive, $d_{1}<0$, therefore the system never reaches the critical surface given by Eq. (3.8). Thus, if the coupling is weaker than the limit given by Eq. (3.9), the slow dynamics of defects can not affect the density dynamics so that the density variable does not slow down. Eq. (3.9) gives the correct relation between the coupling energy and the barrier size of the defects in the case of observed slowing down. In this limit, Eq. (2.65) reduce to

$$
\begin{aligned}
& d_{1}(x, y, \sigma)=-8(y+C)-4\left(\frac{C^{2}}{y}+3 y+8 C\right) \sigma+\mathcal{O}\left(\sigma^{2}\right) \\
& d_{2}(x, y, \sigma)=1+4 y+2(3 y+2 C) \sigma+\mathcal{O}\left(\sigma^{2}\right) .
\end{aligned}
$$


Thus, from Eqs. (3.8) and (3.11), we have the critical condition given in terms of the metastability parameters by

$$
C=\frac{1}{4}\left(1-\frac{2 \lambda+1}{2 \xi \lambda^{2}}\right), \quad y=\frac{1}{4}\left(\frac{1}{\xi \lambda^{2}}-1\right) .
$$

Furthermore, from Eqs. (3.7) and (3.11), we can easily see that the limit $\sigma \rightarrow 0$ can be identified with $\sigma_{0} \rightarrow 0$ if

$$
3\left(\frac{C^{2}}{y}+3 y+8 C\right)=2(1-\lambda)(3 y+2 C) .
$$

Therefore, as indicated in Sec. [1, we have the situation where the stretched dynamics is associated with the weak coupling $(x \rightarrow 0)$ and low barrier $(\sigma \rightarrow 0)$ limit, which is consistent with the separation of the time scales between the density and the defect variables as can be seen from Eq. (2.44). The condition Eq. (3.13), when we use Eq. (3.12), can be interpreted as a relation between the exponent parameter $\lambda$ and the temperature represented by the parameter $\xi$ :

$$
\xi=\frac{1}{\lambda^{2}}\left[1-\frac{3(2 \lambda-1)}{2\left[7+2 \lambda+\sqrt{4 \lambda^{2}+22 \lambda+91}\right]}\right] .
$$

Thus the exponents $a$ and $b$ are given as smooth functions of temperature. (See Fig. 4.) The temperature dependence of the parameters $y$ and $C$ which describe the potential $h(n)$ and its coupling to $\rho$ is given by Eq. (3.12). All the temperature dependences certainly do not show any indication of a special temperature. In the conventional MCT [0].16], the control parameter $\sigma_{0}$ is assumed to have a temperature dependence as $\sigma_{0} \sim T_{0}-T$, which is the origin of the sharp temperature dependences. In this case, however, $\sigma_{0}$ is proportional to $\sigma$ with weakly temperature-dependent coefficient $f(\xi)$ :

$$
\sigma_{0}=f(\xi) \sigma \equiv-\frac{2}{3}[3 y+2 C] \xi \lambda(1-\lambda)^{2} \sigma
$$

We note that $f(\xi)$ vanishes at the lower and upper bounds of $\xi$ which correspond to $\lambda=1$ and $\frac{1}{2}$, respectively. Therefore, the above analysis is not applicable to the region near the two end points of $\xi$. Our basic picture of the observed slowing down of the dynamics is 
that the parameters describing the metastable wells and the coupling, while displaying a smooth temperature dependence, achieve the critical limit given by Eq. (3.9). In this model, the transition is actually controlled by the parameter $\sigma$, which is the size of barrier in the metastable potential.

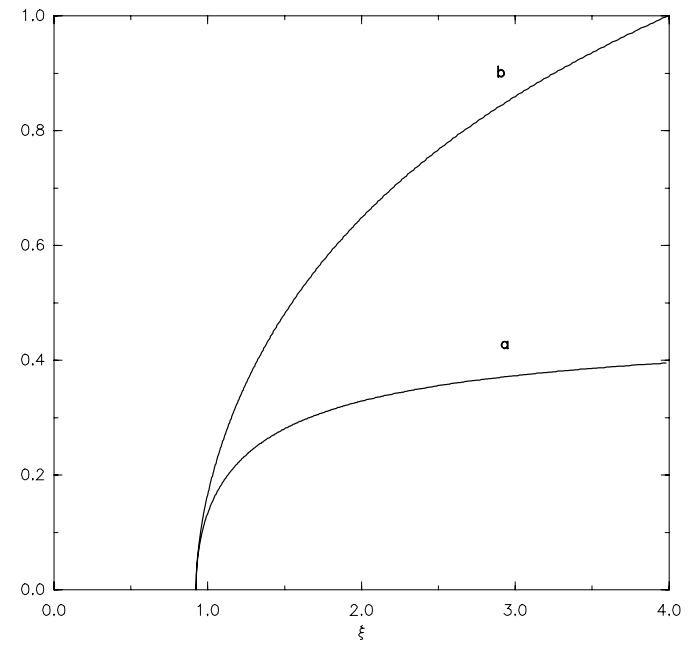

The exponents $a$ and $b$ as functions of temperature represented by $\xi$

FIG. 4

\section{THE MODEL INCLUDING THE DYNAMICS OF DEFECTS}

The approximation, Eq. (3.1), made in the previous section enabled us to treat the model analytically and to obtain the critical condition between the shallow metastable wells and the weak coupling. However, when nonlinear corrections to the bare diffusion coefficient come into play, the constraint Eq. (3.1) must be relaxed and the dynamics of defects should be taken into account. In this section, we analyze the full coupled equations, (2.59) and (2.60) with (2.64) and (2.70). Since the nonlinear couplings in these equations are highly nontrivial, an analytical treatment is very difficult. In the time domain, these equations are coupled integro-differential equations: 


$$
\begin{aligned}
& \ddot{\phi}(t)+d_{0} \dot{\phi}(t)+\Omega_{0}^{2} \phi(t)+\Omega_{0}^{2} \int_{0}^{t} d s H(t-s) \dot{\phi}(s)=0 \\
& \dot{\psi}(t)+\gamma_{v} \psi(t)+\int_{0}^{t} d s G(t-s) \psi(s)=0
\end{aligned}
$$

where

$$
H(t)=c_{1} \psi(t) \phi(t)+c_{2} \phi^{2}(t), \quad G(t)=-\widetilde{\xi} \ddot{\phi}(t) \psi(t)
$$

with the initial conditions $\phi(0)=\psi(0)=1, \dot{\phi}(0)=0$, and the definition $\widetilde{\xi} \equiv\left(\frac{q}{\Lambda}\right)^{2} \xi$. In the following, we integrate them numerically. We fix $d_{0}=\Omega_{0}^{2}=1$ and study the relaxation of the system at fixed wavenumber $q / \Lambda=0.1$ as $\gamma_{v}$ and the MCT coefficients $c_{1}, c_{2}$ change. We use the parametrization Eq. (3.7) for $c_{1}, c_{2}$ and the value of $\xi$ determined from Eq. (3.14).

We find that the system depends in a crucial way on the value of $\gamma_{v}$. When the value of $\gamma_{v}$ is large enough, we expect that the defects quickly diffuse away and only the density fluctuations are important. It is clearly seen in the numerical integration of Eqs. (4.1), (4.2) for fixed $c_{1}, c_{2}$ and $\xi$ in Fig. 5. The defect auto-correlation function $\psi(t)$ decays exponentially for large $\gamma_{v}$. Thus, in the large $\gamma_{v}$ limit, the model essentially reduces to the one originally considered by Leutheusser [7] where only the $c_{2}$ term is present. For smaller values of $\gamma_{v}$, we expect that the system goes into the regime where the result of the previous section applies such that the slow dynamics of defects and the coupling between defects and density fluctuations play an important role in stretched dynamics. We can see from Figs. 5 and 6 that, as the value of $\gamma_{v}$ is decreased, $\psi(t)$ and $\phi(t)$ are more and more stretched. We can not, however, take $\gamma_{v}$ to zero, since for small enough $\gamma_{v}$ such that $\gamma_{v}<\gamma_{v}^{c}$ for some $\gamma_{v}^{c}, \psi(t)$ starts to increase with time and consequently the model becomes unphysical. This surprising result restricts the value of the parameter $\gamma_{v}$ to $\gamma_{v}>\gamma_{v}^{c}$. It is clear that $\psi(t)$ and $\phi(t)$ are stretched most when $\gamma_{v}$ is very close to but still larger than $\gamma_{v}^{c}$. In fact, the time scale for $\psi(t)$ becomes extremely large as $\gamma_{v} \rightarrow \gamma_{v}^{c}$ so that it seems to approach some plateau value $g$ as $t \rightarrow \infty$. We will discuss later that only when $\gamma_{v} \simeq \gamma_{v}^{c}$ is the time scale of $\psi(t)$ much greater than that of $\phi(t), \psi(t)$ is more stretched than $\phi(t)$ and the picture is consistent with the basic picture obtained in the previous section. 
In adddition to $\gamma_{v}$, the system also depends on the MCT coefficients $c_{1}, c_{2}$ such that as one approaches the critical surface in the $\left(c_{1}, c_{2}\right)$ space, the slowing down of $\phi(t)$ is obtained. In general, we expect that due to the defect dynamics, the explicit form of the critical surface will be different from the one given by Eq. (3.7) with $\sigma_{0}=0$. In Fig. 7 , the relaxation of $\phi(t)$ is shown when we change $c_{1}$ and $c_{2}$ but adjust $\gamma_{v}$ such that $\gamma_{v} \simeq \gamma_{v}^{c}$ for each case. We note that in the previous case where $\psi(t) \equiv 1$, the critical slowing down of $\phi(t)$ is achieved when $c_{1}(\lambda)=(2 \lambda-1) / \lambda^{2}, c_{2}(\lambda)=1 / \lambda^{2}$. As can be seen from Fig. 7 , for the case of $\lambda=0.6$, the dynamics of defects influence the system such that at $\left(c_{1}(0.6), c_{2}(0.6)\right)=(0.556,2.778)$, $\phi(t)$ still relaxes with finite time scales. The critical slowing down similar to the one with $\psi(t) \equiv 1$ and $\left(c_{1}, c_{2}\right)=(0.556,2.778)$ occurs at $\left(c_{1}, c_{2}\right) \simeq(0.590,2.714)$ with larger $c_{1}$ and smaller $c_{2}$. We find the similar shift in the critical surface for $\lambda=0.7$ case as in Fig. 8. The relaxation at $\left(c_{1}(0.7), c_{2}(0.7)\right)=(0.816,2.041)$ for the $\psi(t) \equiv 1$ case is similar to the one at $\left(c_{1}, c_{2}\right)=(0.872,1.902)$.

The sequence of the time relaxations of $\phi(t)$ can be most easily seen in a $\log (-t \dot{\phi}(t))$ versus $\log (t)$ plot. In such plot, the power-law and the von-Schweidler relaxations are combined to form a minimum with asymptotic slopes of $-a$ and $b$. The later stage of relaxation with a peak is well fit by the stretched exponential (Fig. 9). As $\gamma_{v} \rightarrow \gamma_{v}^{c}$, we find that the later stage of the relaxation of $\psi(t)$ is also well fit by a stretched exponential. We now use two stretched exponential forms

$$
\phi(t)=f \exp \left(-(t / \tau)^{\beta}\right), \quad \psi(t)=g \exp \left(-\left(t / \tau^{\prime}\right)^{\beta^{\prime}}\right)
$$

in Eqs. (4.1) and (4.2). Since, for $\gamma_{v}$ near $\gamma_{v}^{c}$, the time scale $\tau^{\prime}$ for $\psi(t)$ is much greater than $\tau$ for $\phi(t)$, we can write

$$
\exp \left(-\left(t / \tau^{\prime}\right)^{\beta^{\prime}}\right) \simeq 1-\left(\frac{\tau}{\tau^{\prime}}\right)^{\beta^{\prime}}\left(\frac{t}{\tau}\right)^{\beta^{\prime}} .
$$

We find the following qualitative relations among these parameters from Eqs. (4.1) and (4.2):

$$
\begin{aligned}
& 2^{-\frac{1}{\beta}}=\frac{1-c_{1} g}{c_{2} f}+\frac{c_{1} g}{c_{2} f} \frac{\Gamma\left(\frac{1+\beta^{\prime}}{\beta}\right)}{\Gamma\left(\frac{1}{\beta}\right)}\left(\frac{\tau}{\tau^{\prime}}\right)^{\beta^{\prime}} \\
& \left(\gamma_{v}-\gamma_{v}^{c}\right) \tau=\frac{1}{g \Gamma\left(1+\frac{1}{\beta^{\prime}}\right)}\left(\frac{\tau}{\tau^{\prime}}\right)+f g \beta^{\prime} \tilde{\xi} \Gamma\left(\frac{\beta+\beta^{\prime}-1}{\beta}\right)\left(\frac{\tau}{\tau^{\prime}}\right)^{\beta^{\prime}}
\end{aligned}
$$


Numerically, we find that the second term in the right hand side of Eq. (4.7) is much smaller than the first term. Thus, we can rewrite Eq. (4.7) as

$$
\left(\gamma_{v}-\gamma_{v}^{c}\right) \tau^{\prime} \simeq \frac{1}{g \Gamma\left(1+\frac{1}{\beta^{\prime}}\right)}
$$

which means that $\tau^{\prime}$ diverges as $\left(\gamma_{v}-\gamma_{v}^{c}\right)^{-1}$ when $\gamma_{v} \rightarrow \gamma_{v}^{c}$. By obtaining the parameters $\beta$, $\beta^{\prime}, \tau$ and $\tau^{\prime}$ from explicit curve fits of $\phi(t)$ and $\psi(t)$ using Eq. (4.4) for various values of $\gamma_{v}$ near $\gamma_{v}^{c}$, we find some evidence for this relation (Fig. 10). Without the second term in the right hand side of Eq. (4.6), it gives the expression for $\beta$ of the conventional MCT with $c_{1}$ and $c_{2}(g=1): \beta=-\ln (2) / \ln \left(\left(1-c_{1}\right) / c_{2} f\right)$. However, in the presence of defect dynamics, Eq. (4.6) represents a more complicated expression for $\beta$ which depends on $\beta^{\prime}$ as well as $\gamma_{v}-\gamma_{v}^{c}$. We find again evidence for this relation from our numerical data (Fig. 11).

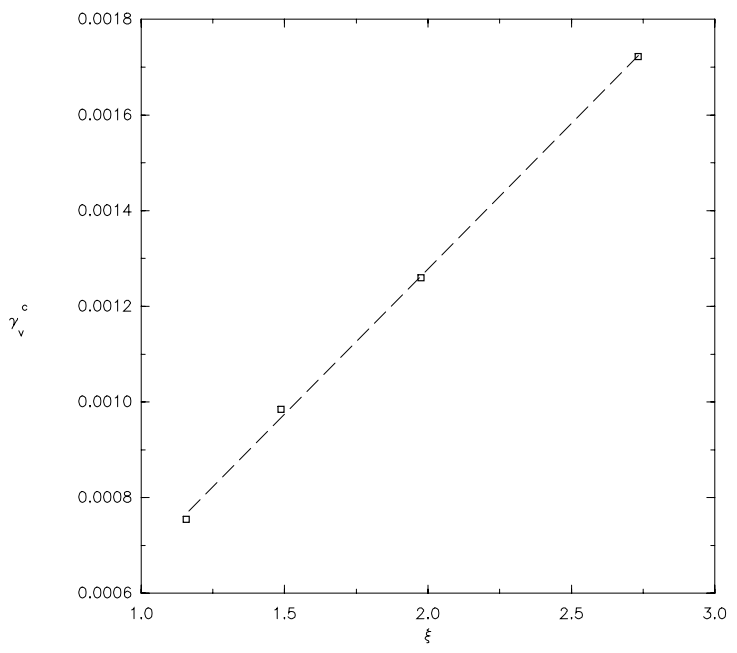

The critical value $\gamma_{v}^{c}$ of $\gamma_{v}$ as a function of the temperature represented by $\xi(\square)$. The dashed line is a linear fit.

FIG. 12

It is clear from these results that the separation of time scales between the defect and the density fluctuations occur only when $\gamma_{v}$ is very close to $\gamma_{v}^{c}$. We find that, as a function of $\xi$, the value of $\gamma_{v}^{c}$ shows a smooth linear temperature dependence (Fig. 12). Under the previous assumption that the slowing down corresponds to the $\sigma \rightarrow 0$ (or $\gamma_{v} \rightarrow 0$ ) limit, we were able 
to determine the temperature dependence of the metastability parameters except for that of $\sigma$ itself. This was done by assuming that the system arranges itself to be on the critical surface. The present analysis of the model including the defect dynamics determines the temperature dependence of the parameter $\gamma_{v} \equiv \sigma y \widetilde{\Gamma}_{v} q^{2}$ when the system is on the critical surface.

\section{DISCUSSION}

It was discovered by Das and Mazenko [11] that the nonhydrodynamic correction due to $\Sigma_{\hat{V} \rho}(\mathbf{q}, \omega)$ included in the representation like Eq. (2.60) cuts off the sharp nature of the ergodic-nonergodic transition. In the presence of the new variable $n$, however, we have a very complicated expression, Eq. (2.53) for the density response function. In order to see the cutoff effect, let the viscosity $\Gamma$ become arbitrarily large. Eq. (2.53) then reduces to

$$
G_{\rho \hat{\rho}}(\mathbf{q}, \omega) \simeq \frac{\omega}{\omega\left(\omega+i q \Sigma_{\widehat{V} \rho}(\mathbf{q}, \omega)\right)+i q \Sigma_{\widehat{V} n}(\mathbf{q}, \omega) \Sigma_{\widehat{n} \rho}(\mathbf{q}, \omega)}
$$

If the self-energies $\Sigma_{\hat{V} n}$ and $\Sigma_{\widehat{n} \rho}$, as well as $\Sigma_{\widehat{V} \rho}$ are set to zero, $G_{\rho \widehat{\rho}}(t)$ reaches a finite value as time $t \rightarrow \infty$. The presence of these self-energies makes $G_{\rho \widehat{\rho}}(t)$ decay slowly and thus provides the cutoff effect. The evaluation of these self-energies in principle can be done to yield complicated expressions in terms of $\phi(t)$ and $\psi(t)$. The analysis including this effect thus can be performed but will be a very difficult task even numerically.

Throughtout this paper, we have concentrated on the time relaxation behavior of the system near the glass transition. At this stage, it is important to note the implication of our model on the temperature dependence of the viscosity: $\eta(T)$. Traditionally, many different expressions have been used to fit the experimentally observed $\eta(T)$. These include the Arrhenius form $\sim \exp (A / T)$, the Vogel-Fulcher form $\sim \exp \left(B /\left(T-T_{V F}\right)\right)$ and the power-law $\sim\left|T-T_{0}\right|^{-\gamma}$. These forms are able to fit the experimental data only over limited temperature ranges. In MCT, the temperature dependence of the viscosity is given by [1]

$$
\eta(T) \sim \tau_{\alpha}(T) \sim\left|\sigma_{0}\right|^{-\gamma}
$$


where $\gamma=\frac{1}{2 a}+\frac{1}{2 b}$. According to conventional MCT, $\sigma_{0}$ is assumed to be proportional to $T_{0}-T$ and $\gamma$ is temperature independent. Thus, the conventional MCT predicts that the viscosity shows a power-law divergence as the temperature approaches $T_{0}$. In our model, the situation is quite different. The temperature dependence of $\sigma_{0}$ is given by Eq. (3.15) without any special temperature. More importantly, $\gamma=1 /(2 a)+1 /(2 b)$ in this model is a function of temperature which is represented by the parameter $\xi$. Thus we have

$$
\eta(\xi)=|f(\xi) \sigma(\xi)|^{-\gamma(\xi)}
$$

where $f(\xi)=\frac{2}{3}[3 y+2 C] \xi \lambda(1-\lambda)^{2}$. Therefore, the temperature dependence of the viscosity is governed mainly by the behavior of the exponent $\gamma(\xi)$ in Eq. (5.3) as a function of $\xi$, i.e. $\log \eta(\xi) \sim \gamma(\xi)$. As shown in Fig. 13, $\gamma(\xi)$ increases as the temperature decreases. We do not expect, however, that Eq. (5.3) can be used directly to fit the experimental data, since $a$ and $b$ as functions of $\xi$ are model-dependent. Especially the rapid increase of $\log \eta(\xi)$ near the lower bound of $\xi$ should not be understood as a physical result as noted in Sec. [III. But this analysis clearly indicates that our model is consistent with the generic feature of the observed $\eta(T)$ which increases with decreasing temperature.

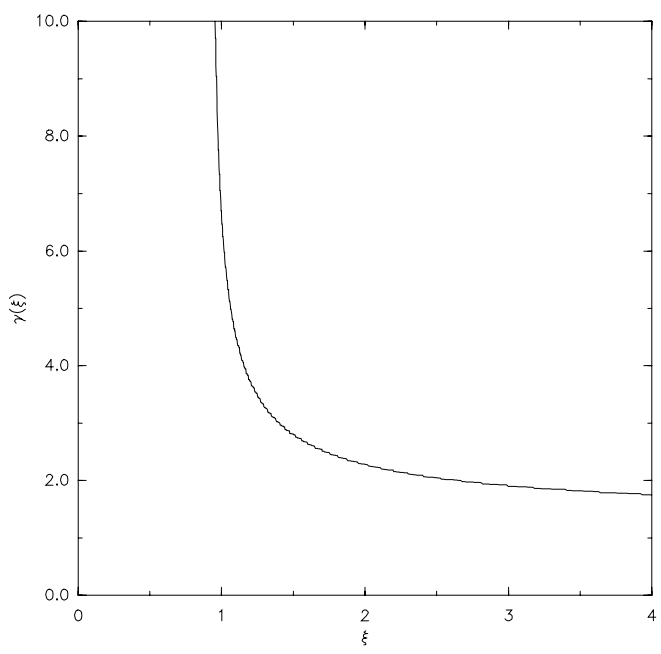

The parameter $\gamma=1 /(2 a)+1 /(2 b)$ as a function of $\xi$

FIG. 13 


\section{APPENDIX: THE MODEL WITH A GENERAL POTENTIAL $h(n)$}

In the present work, we have used an explicit double-well parametrization given by Eq. (2.9) for the potential energy $h(n)$. In this appendix, we show that the similar analysis can be carried out for a general potential $h(n)$ without changing any physical results to the lowest order of the perturbation theory. The only information we use for $h(n)$ is that there exists a metastable defect density $\bar{n}$ such that $h^{\prime}(\bar{n})=0$. Then the potential energy $h(n)$ is described by the following dimensionless parameters:

$$
\mu_{2} \equiv \frac{h^{\prime \prime}(\bar{n}) \bar{n}^{2}}{A \rho_{0}^{2}}, \quad \mu_{3} \equiv \frac{h^{\prime \prime \prime}(\bar{n}) \bar{n}^{3}}{A \rho_{0}^{2}},
$$

and in general, $\mu_{k} \equiv h^{(k)}(\bar{n}) \bar{n}^{k} /\left(A \rho_{0}^{2}\right), k=2,3, \cdots$. Thus, the double-well potential given by Eq. (2.9) is a particular case where

$$
\mu_{2}=\sigma y, \quad \mu_{3}=2 y(1+\sigma), \quad \mu_{4}=6 y
$$

and $\mu_{k}=0$ for $k=5,6, \cdots$.

To the one-loop order, however, only two parameters, $\mu_{2}$ and $\mu_{3}$ are relevant, so that the coefficients $d_{1}$ and $d_{2}$ in Eq. (2.65) are completely described by $x, \mu_{2}$ and $\mu_{3}$. The parameters $\mu_{k}, k=4,5, \cdots$ generate the terms in the action, Eq. (2.33) that contribute to the two-loop and higher order diagrams. This is basically the reason why the physical situation at the one-loop order is well described by the double-well potential, which has two independent parameters $\sigma$ and $y$ beside $\bar{n}$.

We note that Eq. (2.44) was the self-consistent condition that leads to the separation of the time scales between the density and the defect variables. For a general potential it is given by

$$
\left|\frac{\left(\mu_{2}+x\right)^{2}}{\mu_{2}}\right| \ll 1 .
$$

In terms of $\mu_{2}, \mu_{3}$ and $x$, the coefficients $d_{1}$ and $d_{2}$ are given by

$$
\begin{aligned}
& d_{1}\left(x, \mu_{2}, \mu_{3}\right)=-\frac{\mu_{3}^{2} x}{\mu_{2}^{2}}\left(2+\frac{x}{\mu_{2}}\right)-2\left[2\left(\mu_{2}+\mu_{3}\right)+\frac{\mu_{3}^{2} x^{2}}{\mu_{2}^{2}}\right]\left(1+\frac{x}{\mu_{2}}\right)^{2} \\
& d_{2}\left(x, \mu_{2}, \mu_{3}\right)=1-2 \frac{\mu_{3} x}{\mu_{2}}\left(1+\frac{x}{\mu_{2}}\right)+\left[2\left(\mu_{2}+\mu_{3}\right)+\frac{\mu_{3}^{2} x^{2}}{\mu_{2}^{2}}\right]\left(1+\frac{x}{\mu_{2}}\right)^{2}
\end{aligned}
$$


As in the case of the double-well potential, a solution to the critical condition given by Eq. (3.8) which is consistent with Eq. (A3) exists only when we take

$$
x \rightarrow 0, \quad \mu_{2} \rightarrow 0, \quad \frac{x}{\mu_{2}^{2}} \rightarrow \widetilde{C},
$$

for some $\widetilde{C}$. This limit corresponds to the case where the coupling is weak and the potential gets very flat near the metastable defect density $\bar{n}$. We note that $\mu_{3}$ must be finite, otherwise the model reduces to the Leutheusser model [7], where there is no stretching. This indicates that the potential is at least a cubic or higher order polynomial in $(n-\bar{n})$ near $\bar{n}$ in order to have the stretched exponential relaxation. In this limit, we have

$$
\begin{aligned}
& d_{1}\left(x, \mu_{2}, \mu_{3}\right)=-2 \mu_{3}^{2} \widetilde{C}-4 \mu_{3}-\left(4+8 \mu_{3} \widetilde{C}+\mu_{3}^{2} \widetilde{C}^{2}\right) \mu_{2}+\mathcal{O}\left(\mu_{2}^{2}\right) \\
& d_{2}\left(x, \mu_{2}, \mu_{3}\right)=1+2 \mu_{3}+\left(2+2 \mu_{3} \widetilde{C}\right) \mu_{2}+\mathcal{O}\left(\mu_{2}^{2}\right)
\end{aligned}
$$

As done in Sec. [II, we can express the metastability parameters in terms of the temperature $\xi:$

$$
\begin{gathered}
\mu_{3}=\frac{1}{2}\left(\frac{1}{\xi \lambda^{2}}-1\right), \quad \widetilde{C}=\frac{2\left(2-\frac{2 \lambda+1}{\xi \lambda^{2}}\right)}{\left(\frac{1}{\xi \lambda^{2}}-1\right)^{2}}, \\
\xi=\frac{1}{\lambda^{2}}\left[1-\frac{3(2 \lambda-1)}{2\left[2 \lambda+1+\sqrt{4 \lambda^{2}+10 \lambda+13}\right]}\right] .
\end{gathered}
$$

Because of the particular parametrization of $\mu_{2}$ and $\mu_{3}$ in terms of $\sigma$ and $y$, the explicit form for the temperature dependence of the parameters for the double-well potential is slightly different from that of the general potential. (See Fig. 14.) But, any of the physical pictures change from the the double-well potential case. Thus, the double-well parametrization is a very good approximation to the lowest order that provides a concrete physical picture that the observed slowing down corresponds to the weak coupling between the density and the defect variables and the low activation barrier for the metastable defect. 


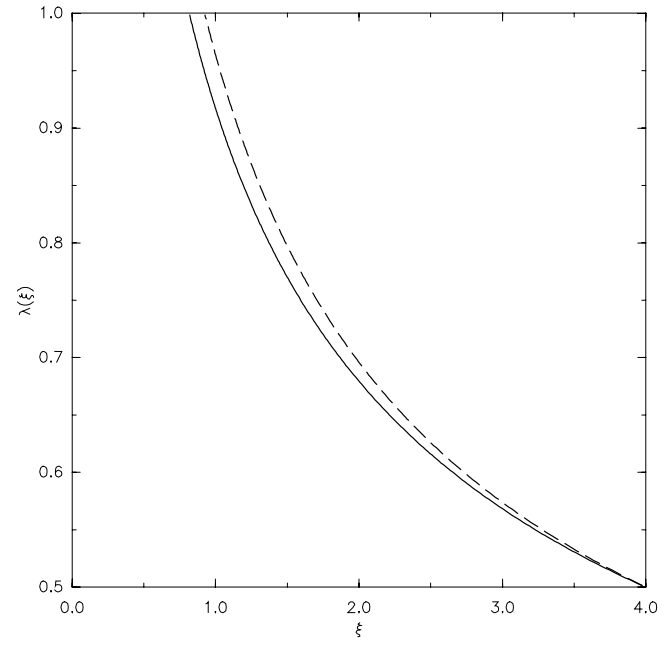

The parameter $\lambda$ as a function of $\xi$ for a general (solid) and the double-well (dashed) potentials FIG. 14

\section{Acknowledgements}

This work was supported by the National Science Foundation Materials Research Laboratory at the University of Chicago. 


\section{REFERENCES}

[1] For a review of theoretical develoment of MCT, see W. Götze, in Liquids, Freezing, and the Glass Transition edited by D. Levesque, J. P. Hansen, and J. Zinn-Justin (New York, Elsevier 1991). See also B. Kim and G. F. Mazenko, Phys. Rev. A. 45, 2393 (1992).

[2] N. J. Tao, G. Li, and H. Z. Cummins, Phys. Rev. Lett. 66, 1334 (1991), G. Li, W. M. Du, X. K. Chen, H. Z. Cummins, and N. J. Tao, Phys. Rev. A 45, 3867 (1992), G. Li, W. M. Du, A. Sakai, and H. Z. Cummins, Phys. Rev. A 46, 3343 (1992).

[3] L. Börjesson, M. Elmroth and L. M. Torell, Chem. Phys. 149, 209 (1990), M. Elmroth, L. Börjesson and L. M. Torell, Phys. Rev. Lett. 68, 79 (1992), D. L. Sidebottom, R. Bergman, L. Börjesson, and L.M. Torrell, Phys. Rev. Lett. 68, 3587 (1992); I. C. Halalay and K. A. Nelson, Phys. Rev. Lett. 69, 636 (1992).

[4] F. Meizei, W. Knaak, and B. Farago, Phys. Rev. Lett. 58, 571 (1987); D. Richter, B. Frick, and B. Farago, Phys. Rev. Lett. 61, 2465 (1988); W. Doster, S. Cusack, and W. Petry, Phys. Rev. Lett. 65, 1080 (1990); W. Petry, E. Bartsch, F. Furuja, M. Kiebel, H. Sillescu, and B. Farago, Z. Phys. B 83, 175 (1991).

[5] A. Schönhals, F. Kremer and E. Schlosser, Phys. Rev. Lett. 67, 999 (1991), A. Schönhals, F. Kremer, A. Hoffmann, E. W. Fischer, and E. Schlosser, Phys. Rev. Lett. 70, 3495 (1993).

[6] P.K. Dixon, L. Wu, S.R. Nagel, B.D. Williams, and J.P. Carini, Phys. Rev. Lett. 65, $1108(1990)$.

[7] E. Leutheusser, Phys. Rev. A 29, 2765 (1984).

[8] S. P. Das, G. F. Mazenko, S. Ramaswamy, and J. Toner, Phys. Rev. Lett. 54, 118 (1985).

[9] More generally one must allow coupling between different wavenumbers. The role of wavenumber will be discussed below. 
[10] W. Götze, Z. Phys. B 56, 139 (1984); B 60, 195 (1985).

[11] S. P. Das and G. F. Mazenko, Phys. Rev. A 34, 2265 (1986).

[12] P. C. Martin, E. D. Rose, and H. A. Siggia, Phys. Rev. A 8, 423 (1973).

[13] U. Deker and F. Haake, Phys. Rev. A 11, 2043 (1975); 12, 1629 (1975). R. Bausch, H. J. Janssen, and H. Wagner, Z. Phys. B 24, 113 (1976); U. Deker, Phys. Rev. A 19, 846 (1979); C. De Dominicis and L. Peliti, Phys. Rev. B 18, 353 (1978); R. V. Jensen, J. Stat. Phys. 25, 183 (1981).

[14] B. Kim, Phys. Rev. A 46, 1992 (1992).

[15] B. Kim and G. F. Mazenko, Phys. Rev. A. 45, 2393 (1992).

[16] U. Bengtzelius, W. Götze and A. Sjölander, J. Phys. C 17, 5915 (1984); W. Götze and L. Sjögren, Z. Phys. B 65, 415 (1987); J. Phys. C. 21, 3407 (1988); J. Phys. Condensed Matter 1, 4183 (1989).

[17] A. Schönhals, F. Kremer, A. Hoffmann, E. W. Fischer, and E. Schlosser, Phys. Rev. Lett. 70, 3495 (1993); D. L. Sidebottom, R. Bergman, L. Börjesson, and L.M. Torrell, Phys. Rev. Lett. 68, 3587 (1992).

[18] H. Z. Cummins, W. M. Du, M. Fuchs, W. Götze, S. Hildebrand, A. Latz, G. Li and N. J. Tao, Phys. Rev. E 47, 4223 (1993).

[19] G. F. Mazenko and J. Yeo, J. Non-Cryst. Solids, to be published.

[20] C. Herring, J. Appl. Phys. 21, 437 (1950); P. C. Martin, O. Parodi, and P. S. Pershan, Phys. Rev. A 6, 2401 (1972). P. D. Fleming and C. Cohen, Phys. Rev. A 13, 500 (1976).

[21] C. Cohen, P. D. Fleming, and J. H. Gibbs, Phys. Rev. A 13, 866 (1976).

[22] L. Sjögren, Z. Phys. B 79, 5 (1990).

[23] S.-k. Ma and G. F. Mazenko, Phys. Rev. B 11, 4077 (1975). 
[24] S.P. Das, Phys. Rev. A 36, 211 (1987).

[25] O. T. Valls and G. F. Mazenko, Phys. Rev. A 46, 7756 (1992); M. Fuchs, I. Hofacker, and A. Latz, Phys. Rev. A 45, 898 (1992).

[26] We could treat more general forms for the potential, but it does not change our basic conclusions very much. See Appendix.

[27] G.F. Mazenko and J. Yeo, J. Stat. Phys. 74, 1017 (1994).

[28] Some quartic terms can also generate the one-loop self-energies. But this contribution is irrelevant to our present discussion. In general, quartic and higher order terms in Eq. (2.33) generate higher-order self-energies.

[29] A recent experiment supporting this factorization property is given in W. van Megan and S. M. Underwood, Phys. Rev. Lett. 70, 2766 (1993). 


\section{Figure Captions}

Fig. 1. A schematic plot of the sequence of relaxation behaviors predicted by MCT; (a) powerlaw decay relaxation $f+A_{1} t^{-a}$; (b) von-Schweidler relaxation $f-A_{2} t^{b}$; (c) primary relaxation $A_{3} e^{-(t / \tau)^{\beta}}$; and $(\mathrm{d})$ exponential relaxation $e^{-\gamma t}$.

Fig. 2. One-loop diagrams contributing to $\Sigma_{\widehat{g} \widehat{g}}(\mathbf{q}, \omega)$.

Fig. 3. One-loop diagrams contributing to $\Sigma_{\widehat{n} \hat{n}}(\mathbf{q}, \omega)$.

Fig. 4. The exponents $a$ and $b$ as functions of temperature represented by $\xi$.

Fig. 5. The defect auto-correlation function $\psi(t)$ for fixed $\left(c_{1}, c_{2}\right)=(0.556,2.778)$ and for various $\gamma_{v}$ : (a) $2.0 \times 10^{-3}$, (b) $1.8 \times 10^{-3}$, (c) $1.75 \times 10^{-3}$, (d) $1.725 \times 10^{-3}$, (e) $1.7225 \times 10^{-3}$, (f) $1.7215 \times 10^{-3}$, (g) $1.721 \times 10^{-3}$, (h) $1.72 \times 10^{-3}$. We can estimate the value of $\gamma_{v}^{c}$ as $1.72 \times 10^{-3}<\gamma_{v}^{c}<1.721 \times 10^{-3}$.

Fig. 6. The density auto-correlation function $\phi(t)$ for fixed $\left(c_{1}, c_{2}\right)=(0.556,2.778)$ and for various $\gamma_{v}$; (a) $2.0 \times 10^{-3}$, (b) $1.8 \times 10^{-3}$, (c) $1.75 \times 10^{-3}$, (d) $1.725 \times 10^{-3}$, (e) $1.7225 \times 10^{-3}$, (f) $1.7215 \times 10^{-3}$, (g) $1.721 \times 10^{-3}$, and (h) $1.72 \times 10^{-3}$.

Fig. 7. The density auto-correlation function $\phi(t)$ for various $\left(c_{1}, c_{2}\right)$; (a) $(0.467,2.944)$, (b) $(0.488,2.905)$, (c) $(0.522,2.842)$, (d) $(0.556,2.778)$, and (e) $(0.590,2.714) . \gamma_{v}$ is adjusted for each case such that $\gamma_{v} \simeq \gamma_{v}^{c}$. (f) $\phi(t)$ at $\left(c_{1}, c_{2}\right)=(0.556,2.778)$ for the case where $\psi(t)=1$. 
Fig. 8. The density auto-correlation function $\phi(t)$ for various $\left(c_{1}, c_{2}\right)$; (a) $(0.719,2.285)$, (b) $(0.779,2.135),(\mathrm{c})(0.816,2.041)$, and $(\mathrm{d})(0.872,1.902) . \quad \gamma_{v}$ is adjusted for each case such that $\gamma_{v} \simeq \gamma_{v}^{c}$. (e) $\phi(t)$ at $\left(c_{1}, c_{2}\right)=(0.816,2.041)$ for the case where $\psi(t)=1$.

Fig. 9. The solid lines are $\log (-t \dot{\phi}(t))$ versus $\log (t)$ plots for $\left(c_{1}, c_{2}\right)=(0.467,2.944)$ and for (a) $\gamma_{v}=1.7225 \times 10^{-3}$ and (b) $\gamma_{v}=1.75 \times 10^{-3}$. The dashed lines are the stretched exponential fits, $\phi(t)=f \exp \left\{-(t / \tau)^{\beta}\right\}$, where (a) $f=0.44, \beta=0.804, \tau=5859$ and (b) $f=0.44, \beta=0.875$, $\tau=4021$.

Fig. 10. The best-fit values of $\left[\tau^{\prime} \Gamma\left(1+\frac{1}{\beta^{\prime}}\right)\right]^{-1}$ for various values of $\gamma_{v}(\square)$. The dashed line is a linear fit.

Fig. 11. $2^{-1 / \beta}$ versus $\frac{\Gamma\left(\frac{1+\beta^{\prime}}{\beta}\right)}{\Gamma\left(\frac{1}{\beta}\right)}\left(\frac{\tau}{\tau^{\prime}}\right)^{\beta^{\prime}}$ plot $(\square)$. The dashed line represents a linear fit.

Fig. 12. The critical value $\gamma_{v}^{c}$ of $\gamma_{v}$ as a function of the temperature represented by $\xi(\square)$. The dashed line is a linear fit.

Fig. 13. The parameter $\gamma=1 /(2 a)+1 /(2 b)$ as a function of the temperature represented by $\xi$.

Fig. 14. The parameter $\lambda$ as a function of $\xi$ for a general (solid) and the double-well (dashed) potentials. 